\title{
Cohomology of topological groups with applications to the Weil group
}

\begin{abstract}
M. Flach
Abstract

We establish various properties of the definition of cohomology of topological groups given by Grothendieck, Artin and Verdier in SGA4, including a Hochschild-Serre spectral sequence and a continuity theorem for compact groups. We use these properties to compute the cohomology of the Weil group of a totally imaginary field, and of the Weilétale topology of a number ring recently introduced by Lichtenbaum (both with integer coefficients).
\end{abstract}

\section{Introduction}

Cohomology of topological groups has been a popular subject with many writers. Soon after the introduction of cohomology of groups by Eilenberg and MacLane [EM47], cohomology of profinite groups was defined by Tate (see the bibliographical notes in [Ser65, ch. I]) and cohomology of Lie groups by van Est [vEs53] and Hochschild and Mostow [HM62]. In both cases the definition is made by simply using continuous instead of arbitrary cochains in the standard complex. A definition using measurable cochains is due to Moore [Moo64] and categorical definitions are due to Wigner [Wig73], Segal [Seg70], Grothendieck, Artin and Verdier [SGA4] and Lichtenbaum [Lic04].

In the first part of this paper we work out the definition of Grothendieck et al. in [SGA4] and show that it has a number of agreeable properties. In particular, there is a Hochschild-Serre spectral sequence under quite general assumptions and a continuity theorem for compact groups which generalizes a well known statement for profinite groups. The definitions of [SGA4] and Lichtenbaum turn out to be equivalent and agree with the continuous cochain definition, as well as those of the other authors mentioned above, in many instances.

Our interest in topological group cohomology derives from Lichtenbaum's recent intriguing paper [Lic04] in which he defines a Weil-étale topos $\bar{Y}_{W}$, for $Y=\operatorname{Spec}\left(\mathcal{O}_{F}\right)$ the spectrum of the ring of integers in a number field $F$, and shows that the groups $H_{c}^{i}\left(Y_{W}, \mathbb{Z}\right), i \leqslant 3$, are related to the Dedekind Zeta-function of $F$ at $s=0$. These computations are made by reduction to the topological group cohomology of the Weil group $W_{F}$ of $F$. For a complete relationship with the Zeta-function one would expect $H_{c}^{i}\left(Y_{W}, \mathbb{Z}\right)$ to vanish for $i \geqslant 4$ but, using the techniques developed in the first part of this paper, we show in the second part that $H^{i}\left(W_{F}, \mathbb{Z}\right)$ and as a consequence also $H_{c}^{i}\left(Y_{W}, \mathbb{Z}\right)$ are in fact infinitely generated abelian groups for even $i \geqslant 4$ (and totally imaginary $F$ ). We also show that $H^{i}\left(W_{F}, \mathbb{Z}\right)=0$ for odd $i$, a result deduced in [Lic04] for $i=3$ from Rajan's paper [Raj04].

This paper owes its existence entirely to the beautiful ideas of Lichtenbaum in [Lic04] and is part of an on-going project with Thomas Geisser exploring the Weil-étale topology both for schemes over finite field and in characteristic zero. What we do below is to a large extent only a reworking

Received 7 October 2006, accepted in final form 1 October 2007.

2000 Mathematics Subject Classification 14F20 (primary), 11G40, 18F10, 22A99 (secondary).

Keywords: cohomology, topological groups, Weil group.

The author is supported by grant DMS-0401403 from the National Science Foundation.

This journal is (C) Foundation Compositio Mathematica 2008. 


\section{FLACH}

of [Lic04] using somewhat more machinery. Since the theory of Weil-étale cohomology is still in its infancy it might be useful to collect such machinery. We hope the properties of topological group cohomology we collect in $\S \S 2$ to 9 are also of some interest independent of Weil-étale cohomology.

\section{The 'gros topos' of topological spaces}

We follow the foundational conventions of [SGA4] based on the axiom of the existence of universes in set theory. Following [SGA4, IV.2.5] we let Top be the category of topological spaces (which are elements of a given universe $\mathbb{U}$ ) and $J_{\text {open }}$ the open covering topology on the category Top. The pair (Top, $\left.J_{\text {open }}\right)$ is a $\mathbb{V}$-site and the category $\mathcal{T}=\operatorname{Sh}\left(\right.$ Top, $\left.J_{\text {open }}\right)$ of sheaves of sets a $\mathbb{V}$-topos where $\mathbb{V}$ is a universe so that $\mathbb{U} \in \mathbb{V}$. From now on we suppress any mention of universes and remark, as in [SGA4], that any concrete computations we do later can also be justified by replacing Top with the subcategory of spaces of appropriately bounded cardinality.

Lemma 1. Let $J_{l s}$ be the topology on Top generated by all families $\left\{f_{\iota}: X_{\iota} \rightarrow X\right\}$ of local section coverings, i.e. so that for all $x \in X$ there is an open neighborhood $x \in U$, an index $\iota$ and a continuous $s: U \rightarrow X_{\iota}$ so that $f_{\iota} s=\mathrm{id}_{U}$. Then $J_{l s}=J_{\text {open }}$.

Proof. Clearly $J_{\text {open }} \subseteq J_{l s}$. Every covering family for $J_{l s}$ can be refined by a local section cover because local section covers are a pretopology [SGA4, II, Proposition 1.4]. By definition a local section cover can be refined by an open cover. So every covering family for $J_{l s}$ lies in $J_{\text {open }}$ [SGA4, II, 1.1.1] and we have $J_{l s}=J_{\text {open }}$.

Lemma 2. An object $\mathcal{F}$ of $\mathcal{T}=\operatorname{Sh}\left(\right.$ Top, $\left.J_{\text {open }}\right)$ can be given by a collection of sheaves $\mathcal{F}_{X}$ for each topological space $X$ (i.e. functors $\mathcal{F}_{X}: \operatorname{Ouv}(X)^{\mathrm{op}} \rightarrow$ Set from the category of open sets with the sheaf property for open coverings) together with morphisms $\tau_{f}: f^{*} \mathcal{F}_{X} \rightarrow \mathcal{F}_{Y}$ for each continuous map $f: Y \rightarrow X$ and transition isomorphisms for a composite $Z \rightarrow Y \rightarrow X$ so that $\tau_{f}$ is an isomorphism if $f$ is an open embedding.

Proof. This is [SGA4, IV.4.10.3].

Let

$$
y: \text { Top } \rightarrow \mathcal{T}
$$

be the Yoneda embedding which is fully faithful (since $J_{\text {open }}$ is subcanonical) and commutes with arbitrary limits. We shall be interested in instances where $y$ also commutes with certain colimits.

Recall that a diagram

$$
X_{1} \rightrightarrows X_{0}
$$

in a category $\mathbf{C}$ is an equivalence relation on $X_{0}$ if

$$
\operatorname{Hom}_{\mathbf{C}}\left(U, X_{1}\right) \rightarrow \operatorname{Hom}_{\mathbf{C}}\left(U, X_{0}\right) \times \operatorname{Hom}_{\mathbf{C}}\left(U, X_{0}\right)
$$

is the graph of an equivalence relation for all $U$, and that this equivalence relation is called effective if the colimit $X$ of (1) exists and $X_{1} \cong X_{0} \times_{X} X_{0}$.

Lemma 3. Suppose that $X_{1}$ is an effective equivalence relation on $X_{0}$ and that $X_{0} \rightarrow X$ is a covering morphism for a subcanonical topology $J$ on a category $\mathbf{C}$ with finite limits. Then the Yoneda embedding

$$
y: \mathbf{C} \rightarrow \operatorname{Sh}(\mathbf{C}, J)
$$

preserves the colimit of (1) (and $y\left(X_{1}\right)$ is an effective equivalence relation on $y\left(X_{0}\right)$ ).

Proof. This is [SGA3, IV, Lemme 4.6.5] applied to the family (M) of covering morphisms for the topology $J$. 


\section{COHOMOLOGY OF TOPOLOGICAL GROUPS}

Any homomorphism $G_{1} \stackrel{i}{\rightarrow} G_{2}$ of group objects in a category $\mathbf{C}$ (with finite limits) which is a monomorphism gives rise to an equivalence relation

$$
G_{1} \times G_{2} \rightrightarrows G_{2}
$$

on $G_{2}$ (where the arrows are given by projection and multiplication). The sequence

$$
1 \rightarrow G_{1} \stackrel{i}{\rightarrow} G_{2} \stackrel{p}{\rightarrow} G_{3} \rightarrow 1
$$

is called exact if (2) is an effective equivalence relation with colimit $G_{3}$. If $G_{3}$ is also a group object and $p$ a group homomorphism then the equivalence relation (2) is compatible with the group structure, in the sense that the multiplication map $\mu: G_{2} \times G_{2} \rightarrow G_{2}$ extends to a map

$$
\mu^{\prime}:\left(G_{1} \times G_{2}\right) \times\left(G_{1} \times G_{2}\right) \rightarrow\left(G_{1} \times G_{2}\right)
$$

commuting with both arrows in (2). Equivalently, the conjugation action $c: G_{2} \times G_{2} \rightarrow G_{2}$ restricts to an action $c: G_{2} \times G_{1} \rightarrow G_{1}$, i.e. $G_{1}$ is normal. An immediate computation with fiber products and the multiplication map then shows that the condition $G_{1} \times G_{2} \cong G_{2} \times{ }_{G_{3}} G_{2}$ is equivalent to $G_{1} \cong * \times_{G_{3}} G_{2}$, i.e. $G_{1}$ is the kernel of $p$ in the usual sense. If $\mathbf{C}$ is an abelian category (in which case all objects are canonically group objects) then the colimit of $(2)$ and of $G_{1} \rightrightarrows G_{2}$ (inclusion and zero map) agree, and we recover the usual notion of exactness.

Lemma 4. Suppose that (3) is an exact sequence of topological groups, i.e. $G_{3}$ is homeomorphic via $p$ to the coset space $G_{2} / G_{1}$ with the quotient topology and $G_{1}$ is homeomorphic to the kernel of $p$ with its subspace topology. Suppose in addition that $p$ is a local section cover, i.e. a covering morphism for $J_{\text {open }}$. Then the image of the sequence (3) under $y:$ Top $\rightarrow \mathcal{T}$ is exact.

Proof. This is immediate from Lemma 3.

Lemma 5. Suppose that $X=\coprod_{\iota \in I} X_{\iota}$ is a coproduct in a category $\mathbf{C}$ with a subcanonical topology $J$ so that the following hold.

(a) The natural maps $X_{\iota} \rightarrow X$ are monomorphisms.

(b) If $\iota \neq \kappa$ then $y\left(X_{\iota} \times_{X} X_{\kappa}\right)$ is an initial object of $\operatorname{Sh}(\mathbf{C}, J)$.

(c) The family $\left\{X_{\iota} \rightarrow X\right\}$ is a covering for the topology $J$.

Then the Yoneda embedding

$$
y: \mathbf{C} \rightarrow \operatorname{Sh}(\mathbf{C}, J)
$$

preserves this coproduct, i.e. $y X=\coprod_{\iota \in I} y X_{\iota}$.

Proof. This is [SGA4, II, Corollary 4.6.2].

Corollary 1. The Yoneda embedding $y:$ Top $\rightarrow \mathcal{T}$ commutes with coproducts.

Proof. Coproducts in Top are the disjoint union of the underlying sets and a subset $U \subseteq X$ is open if and only if $U \cap X_{\iota} \subseteq X_{\iota}$ is open for all $\iota \in I$. So clearly parts (a) and (c) are satisfied for $J_{\text {open. }}$. If $\iota \neq \kappa$ then $X_{\iota} \times_{X} X_{\kappa}$ is the empty topological space $\emptyset$. The empty sieve is an open cover of $\emptyset$, i.e. belongs to $J_{\text {open }}(\emptyset)$. By [SGA4, II, Corollary 4.6.1 1] (or Lemma 5 applied to the empty set $I$ ) $y \emptyset=y\left(X_{\iota} \times_{X} X_{\kappa}\right)$ is an initial object of $\mathcal{T}=\operatorname{Sh}\left(\right.$ Top, $\left.J_{\text {open }}\right)$. Hence, part (b) is also satisfied.

The following result might not be optimal but is sufficient for our applications.

Proposition 2.1. Suppose that $G_{1}$ is a closed subgroup of $G_{2}$ and that either $G_{1}$ is a Lie group and $G_{2}$ Hausdorff or $G_{2}$ compact with countable basis and $G_{3}:=G_{2} / G_{1}$ profinite. Then $p: G_{2} \rightarrow G_{3}$ is a local section cover. In particular the image of the sequence (3) is exact in $\mathcal{T}$. 


\section{FLACH}

Proof. If $G_{2} / G_{1}$ is profinite it is zero-dimensional in the sense of Lebesgue, i.e. every cover has a refinement by a disjoint cover (see [Lic04, Corollary 2.4] for this argument). Since $G_{2}$ is compact with countable basis it is metrizable [Bou89, ch. IX, §3.1, Proposition 1] and complete [Bou89, ch. II, $\S 4.1$, Proposition 1]. The same properties hold for the closed subgroup $G_{1}$. Then [Mic56, Corollary 1.3] applies for $n=-1$ and yields that $p$ is a local section cover. If $G_{1}$ is a Lie group then [Ser49, Theorem 1] shows that $p$ is a local section cover. Lemma 4 shows the remaining statement.

We end this section by introducing further terminology which will be used in the rest of the paper. We call a geometric morphism $f: \mathcal{E} \rightarrow \mathcal{E}^{\prime}$ of topoi totally acyclic if $R^{i} f_{*} \mathcal{A}=0$ for $i>0$ and all abelian group objects $\mathcal{A}$ of $\mathcal{E}$. We call $f$ acyclic if $R^{i} f_{*} f^{*} \mathcal{A}^{\prime}=0$ for $i>0$ and all abelian group objects $\mathcal{A}^{\prime}$ of $\mathcal{E}^{\prime}$, and if in addition $f$ is connected (i.e. the adjunction $\mathcal{A}^{\prime} \rightarrow f_{*} f^{*} \mathcal{A}^{\prime}$ is an isomorphism). Hence if $f$ is totally acyclic and connected then $f$ is also acyclic. 'Acyclic' seems to be standard terminology whereas we have borrowed the terminology 'totally acyclic' from [SGA4, V, Problem 4.14].

We call $f$ a homotopy equivalence if there is a geometric morphism $g: \mathcal{E}^{\prime} \rightarrow \mathcal{E}$ and natural transformations $\operatorname{id}_{\mathcal{E}^{\prime}} \rightarrow g^{*} f^{*}$ and $f^{*} g^{*} \rightarrow \mathrm{id}_{\mathcal{E}}$. For example, the canonical morphism $f: \mathcal{T} \rightarrow$ Set is a totally acyclic homotopy equivalence [MM92, IV.10], [SGA4, IV.4.10] since in that case $f_{*}=g^{*}$ is both left and right exact.

Given a set $S$ we define a generalized topology on $S$ to be a sheaf $\mathcal{F}$ on Top for the open covering topology so that $\mathcal{F}(\{*\})=S$. If $\mathcal{F}$ is represented by a topological space $X$ then

$$
S=\mathcal{F}(\{*\})=\operatorname{Hom}_{\text {Top }}(\{*\}, X)=\operatorname{Hom}_{\text {Set }}(\{*\}, X)=X,
$$

and so a topology on $S$ in the usual sense gives a generalized topology.

A generalized topology seems to be a rather weak structure but it is carried by the cohomology groups defined in the next section.

We follow the conventions and definitions of Bourbaki [Bou89] concerning set-theoretic topology. In particular, compact and locally compact topological spaces are assumed to be Hausdorff.

\section{Definition of topological group cohomology}

For any category $\mathbf{C}$ and group object $G$ in $\mathbf{C}$ we denote by $B_{\mathbf{C}} G$ the category of $G$-objects in $\mathbf{C}$ (objects $X$ together with an action $G \times X \rightarrow X$ ).

The functor $y$ : Top $\rightarrow \mathcal{T}$ commutes with arbitrary limits. Any topological group $G$ therefore gives rise to a group object $y G$ of $\mathcal{T}$ and we define the classifying topos of $G$ as

$$
B G:=B_{\mathcal{T}}(y G),
$$

the category of objects $\mathcal{F}$ of $\mathcal{T}$ equipped with an action $y G \times \mathcal{F} \rightarrow \mathcal{F}$. By [SGA4, IV.2.4] the category $B G$ is again a topos.

Given an abelian group object $\mathcal{A}$ of $B G$ (i.e. a sheaf of abelian groups $\mathcal{A}$ on Top, together with group actions $\operatorname{Hom}_{\text {Top }}(U, G) \times \mathcal{A}(U) \rightarrow \mathcal{A}(U)$ functorial in the topological space $\left.U\right)$ we define as usual

$$
H^{i}(G, \mathcal{A}):=\left(R^{i} \Gamma\right)(\mathcal{A}),
$$

where $\Gamma(\mathcal{A})=\mathcal{A}^{G}:=\operatorname{Hom}_{B G}(*, \mathcal{A})$ is the global section functor. If $A$ is a topological $G$-module we set

$$
H^{i}(G, A):=H^{i}(G, y A) .
$$

This is just a special case of the definition of cohomology in an arbitrary topos $\mathcal{E}$ which in turn is 


\section{COHOMOLOGY OF TOPOLOGICAL GROUPS}

a special case of the formation of higher direct images with respect to a morphism of topoi $\mathcal{E} \rightarrow \mathcal{E}^{\prime}$ (namely the case of the unique morphism $\mathcal{E} \rightarrow$ Set). We have a factorization of morphisms of topoi

$$
B G \stackrel{e_{G}}{\longrightarrow} B *=\mathcal{T} \stackrel{u}{\rightarrow} \text { Set, }
$$

where $e_{G}$ is the morphism of classifying topoi induced by the unique homomorphism of group objects $y G \rightarrow *$ in $\mathcal{T}$. So $e_{G}^{*} \mathcal{A}$ is $\mathcal{A}$ with trivial $y G$-action and $e_{G, *} \mathcal{A}=\underline{\operatorname{Hom}}_{G}(*, \mathcal{A})$ where $\underline{\operatorname{Hom}}_{G}(-,-):=$ $\underline{\operatorname{Hom}}_{y G}(-,-)$ is the internal Hom-object of $\mathcal{T}$ defined in [MM92, VII.3]. If $\mathcal{T}$ is an arbitrary topos and $\mathcal{G}$ a group object of $\mathcal{T}$ then by [MM92, VII.3, (8)] one has an adjunction

$$
\operatorname{Hom}_{B_{\mathcal{T}} \mathcal{G}}(X \times Z, Y) \cong \operatorname{Hom}_{\mathcal{T}}\left(X, \underline{\operatorname{Hom}}_{\mathcal{G}}(Z, Y)\right)
$$

in the variables $X$ of $\mathcal{T}$ and $Y, Z$ of $B_{\mathcal{T}} \mathcal{G}$. As remarked above, $u$ is totally acyclic, i.e. $u_{*}$ is exact. Hence the Leray spectral sequence for the composite $u \circ e$ degenerates and gives a generalized topology on the groups $H^{i}(G, \mathcal{A})=u_{*}\left(R^{i} e_{G, *}\right)(\mathcal{A})=\left(R^{i} e_{G, *}\right)(\mathcal{A})(\{*\})$.

Lemma 6. Let $G$ be a topological group and

$$
0 \rightarrow A_{1} \stackrel{i}{\rightarrow} A_{2} \stackrel{p}{\rightarrow} A_{3} \rightarrow 0
$$

an exact sequence of topological $G$-modules so that $p$ is a local section cover. Then there is a long exact cohomology sequence

$$
\cdots \rightarrow H^{i}\left(G, A_{1}\right) \rightarrow H^{i}\left(G, A_{2}\right) \rightarrow H^{i}\left(G, A_{3}\right) \rightarrow H^{i+1}\left(G, A_{1}\right) \rightarrow \cdots .
$$

Proof. By Lemma 4 the sequence

$$
0 \rightarrow y A_{1} \stackrel{i}{\rightarrow} y A_{2} \stackrel{p}{\rightarrow} y A_{3} \rightarrow 0
$$

is exact in $\mathrm{Ab}(\mathcal{T})$ and hence in $\mathrm{Ab}(B G)$.

\section{Comparison to the definition of Lichtenbaum}

If $X$ is a $G$-space, i.e. an object of $B_{\text {Top }} G$, then $y X$ is an object of $B G$ and the functor

$$
i: B_{\text {Top }} G \rightarrow B G, \quad X \mapsto y X
$$

is fully faithful. On the category $B_{\mathrm{Top}} G$ we consider the Grothendieck topology $J_{l s}$ generated by all covering families $\left\{X_{\iota} \rightarrow X\right\}$ with local sections in Top. In other words, the topology $J_{l s}$ on $B_{\text {Top }} G$ is induced by the topology $J_{l s}$ on Top via the natural forgetful functor $B_{\text {Top }} G \rightarrow$ Top. For the notion of induced topology we refer to [SGA4, III.3.1].

Proposition 4.1. The topology on $B_{\mathrm{Top}} G$ induced by the canonical topology on $B G$ via the functor $i$ coincides with $J_{l s}$. Moreover, the set of objects of $B_{\text {Top }} G$ is a generating family for the category $B G$.

Proof. There is a commutative diagram of functors

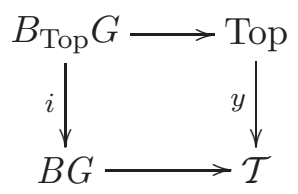

where the horizontal arrows are the forgetful functors. Since $\mathcal{T}$ is a topos, the forgetful functor has a right adjoint $Y \mapsto Y^{G}$ (in fact the forgetful functor is the pullback for an essential morphism of topoi $\mathcal{T} \rightarrow B G)$. Together with the fact that the forgetful functor is faithful this implies that a family $\left\{X_{\iota} \rightarrow X\right\}$ in $B G$ is epimorphic if and only if it is epimorphic in $\mathcal{T}$. Since the covering families for the canonical topology of a topos are precisely the epimorphic families, we deduce from [SGA4, III, Corollary 3.3] that the topology induced on $B G$ from $J_{\text {can }}$ on $\mathcal{T}$ is $J_{\text {can }}$. 


\section{FLACH}

On the other hand, the topology induced on Top from $J_{\text {can }}$ on $\mathcal{T}$ is $J_{\text {open }}=J_{l s}$ by [SGA4, IV, Corollary 1.2.1] and Lemma 1. The first part of the lemma then follows from the commutativity of $(6)$.

Given a category $\mathbf{C}$ with group object $G$ the forgetful functor $B_{\mathbf{C}} G \rightarrow \mathbf{C}$ has a left adjoint

$$
\operatorname{Hom}_{\mathbf{C}}(X, Y) \cong \operatorname{Hom}_{B_{\mathbf{C}} G}(G \times X, Y) .
$$

Here $G$ acts on $G \times X$ via the first factor. It follows that if $\left\{X_{\iota}\right\}$ is a generating family for $\mathbf{C}$ then $\left\{G \times X_{\iota}\right\}$ is a generating family for $B_{\mathbf{C}} G$. If $\mathcal{E}=\operatorname{Sh}(\mathbf{C}, J)$ is a Grothendieck topos then ob(C), or rather $\left\{a y_{\mathbf{C}}(C), C \in \mathrm{ob}(\mathbf{C})\right\}$, is a generating family for $\mathcal{E}$ [SGA4, II, Corollary 4.1.1]. So if $G$ is a group object in $\mathbf{C}$ then $\left\{a y_{\mathbf{C}}(G \times C), C \in \mathrm{ob}(\mathbf{C})\right\}$ is a generating family for $B_{\mathcal{E}}\left(a y_{\mathbf{C}}(G)\right)$. Hence so is $\left\{a y_{\mathbf{C}}(X), X \in \mathrm{ob}\left(B_{\mathbf{C}} G\right)\right\}$.

Corollary 2. The functor

$$
B G \rightarrow \operatorname{Sh}\left(B_{\text {Top }} G, J_{l s}\right) ; \quad \mathcal{F} \mapsto \operatorname{Hom}_{B G}(i(-), \mathcal{F})
$$

is an equivalence of categories. In other words, the Lichtenbaum site $\left(B_{\mathrm{Top}} G, J_{l s}\right)$ of $[\mathrm{Lic04}, \S 1]$ is a site for the classifying topos BG considered in [SGA4].

Proof. This is [SGA4, IV, Corollary 1.2.1].

Corollary 3. Let $G \times$ Top be the full subcategory of $B_{\text {Top }} G$ consisting of spaces of the form $G \times X$ with $G$ acting on the first factor. Then $\operatorname{Sh}\left(G \times \operatorname{Top}, J_{l s}\right)=\operatorname{Sh}\left(B_{\text {Top }} G, J_{l s}\right)$.

Proof. The proof of Proposition 2 shows that $\{y(G \times X), X \in \mathrm{ob}(\mathrm{Top})\}$ is a generating family of $B G$ and that the topology induced by $J_{\text {can }}$ coincides with $J_{l s}$. The result then follows again from [SGA4, IV, Corollary 1.2.1].

\section{Comparison to continuous cochains}

This section just works out an exercise in [SGA4, V, Exercice 3.6] and reproduces the results of Lichtenbaum in [Lic04, §2]. For any topos $\mathcal{T}$ and group object $\mathcal{G}$ of $\mathcal{T}$ denote by $E \mathcal{G}$ the object of $B_{\mathcal{T}} \mathcal{G}$ consisting of $\mathcal{G}$ with its natural left $\mathcal{G}$-action.

LEMMA 7.

(a) For any object $\mathcal{X}$ of $\mathcal{T}$ there is an equivalence of topoi

$$
B_{\mathcal{T}} \mathcal{G} /(E \mathcal{G} \times \mathcal{X}) \rightarrow \mathcal{T} / \mathcal{X}
$$

sending $Y \rightarrow E \mathcal{G} \times \mathcal{X}$ to $Y \times_{E \mathcal{G} \times \mathcal{X}}\{*\} \times \mathcal{X}$ and $Z \rightarrow \mathcal{X}$ to $E \mathcal{G} \times Z \rightarrow E \mathcal{G} \times \mathcal{X}$.

(b) If $\mathcal{X}$ is an object of $B_{\mathcal{T}} \mathcal{G}$ then $E \mathcal{G} \times \mathcal{X}$ with action on the first factor is isomorphic to $E \mathcal{G} \times \mathcal{X}$ with diagonal action.

Proof. Both parts follow from the axioms for group objects and $\mathcal{G}$-objects in a category. The isomorphism in part (b) is given by $\left(p_{1}, \mu\right)$ where $p_{1}: E \mathcal{G} \times \mathcal{X} \rightarrow E \mathcal{G}$ (respectively $\mu: E \mathcal{G} \times \mathcal{X} \rightarrow \mathcal{X}$ ) is the projection (respectively action map).

For $\mathcal{T}=\operatorname{Sh}\left(\right.$ Top, $\left.J_{\text {open }}\right)$ and a topological group $G$ we write $E G$ for $E(y G)$. The map $E G \rightarrow *$ in $B G$ is an epimorphism because it is the image of the local section cover $G \rightarrow\{*\}$ in Top under $y$. Hence the standard simplicial object of this epimorphism

$$
\cdots \rightarrow E G \times E G \times E G \rightarrow E G \times E G \rightarrow E G
$$

gives rise to a Cartan-Leray spectral sequence [SGA4, V, Corollary 3.3]

$$
H^{p}\left(H_{\mathcal{T}}^{q}\left(E G^{\bullet}, \mathcal{A}\right)\right) \Rightarrow H^{p+q}(G, \mathcal{A})
$$




\section{COHOMOLOGY OF TOPOLOGICAL GROUPS}

since by Lemma 7 we have, for $n \geqslant 0$,

$$
H_{B G}^{q}\left(E G^{n+1}, \mathcal{A}\right)=H_{B G / E G^{n+1}}^{q}\left(*, E G^{n+1} \times \mathcal{A}\right)=H_{\mathcal{T} / E G^{n}}^{q}\left(*, E G^{n} \times \mathcal{A}\right)=H_{\mathcal{T}}^{q}\left(E G^{n}, \mathcal{A}\right) .
$$

Proposition 5.1. If $G$ is a topological group and $\mathcal{A}$ an abelian sheaf on Top with $y G$-action so that the sheaf cohomology $H_{\mathcal{T}}^{q}\left(G^{n}, \mathcal{A}\right)$ of the space $G^{n}$ vanishes for $n, q>0$ then $H^{p}(G, \mathcal{A})$ is the cohomology of a complex

$$
\mathcal{A}(\{*\}) \rightarrow \Gamma_{\mathcal{T}}(G, \mathcal{A}) \rightarrow \Gamma_{\mathcal{T}}(G \times G, \mathcal{A}) \rightarrow \cdots,
$$

which agrees with the standard continuous cochain complex if $\mathcal{A}$ is representable by a topological G-module A.

Proof. This is immediate from (7).

Corollary 4. For a topological abelian group $A$ with trivial $G$-action we have $H^{1}(G, A)=$ $\operatorname{Hom}_{\text {cont }}(G, A)$.

Proof. (See also [Lic04, Corollary 1.5].) This follows from $(7)$ and the fact that $H_{\mathcal{T}}^{q}(\{*\}, \mathcal{A})=0$ for $q>0$.

Proposition 5.2. If $G$ is a topological group so that the spaces $G^{n}$ are locally contractible, and $A$ is a discrete abelian group with trivial $G$-action, then $H^{p}(G, A)$ coincides with the singular cohomology of the topological classifying space $\mathbf{B} G$ with coefficients in $A$.

Proof. (See also [SGA4, V, Exercice 3.6, 3].) The topological classifying space $\mathbf{B} G$ is the geometric realization of the simplicial topological space $G^{\bullet}$, and if the spaces $G^{n}$ are locally contractible then $H_{\mathcal{T}}^{q}\left(G^{n}, y A\right)$ coincides with singular cohomology. The natural map from the spectral sequence $(7)$ to the spectral sequence for the cohomology of a simplicial space [Seg70, Proposition 5.1] is then an isomorphism on initial terms, hence an isomorphism on the end terms.

\section{Functoriality in $G$}

Any continuous homomorphism of topological groups $f: G \rightarrow G^{\prime}$ induces a homomorphism of group objects yf : y $G_{1} \rightarrow y G_{2}$ in $\mathcal{T}$, and we denote by $B f: B G \rightarrow B G^{\prime}$ the induced geometric morphism of topoi [SGA4, IV.4.5.1].

Proposition 6.1 (Shapiro lemma). If $f: U \rightarrow G$ is the inclusion of an open subgroup of finite index then $B f$ is totally acyclic.

Proof. Since $U$ is an open subgroup we have $G=\coprod_{s \in G / U} U s$ both in the category Top and in the category $B_{\text {Top }} U$. By Lemma 1, $y:$ Top $\rightarrow \mathcal{T}$ preserves this coproduct and since the forgetful functors in (6) create colimits we also have $E G=y G=\coprod_{s \in G / U} y(U s)$ in $B U$. For any abelian group object $\mathcal{A}$ of $B U$ we have

$$
(B f)_{*} \mathcal{A}=\underline{\operatorname{Hom}}_{U}(E G, \mathcal{A})=\underline{\operatorname{Hom}}_{U}\left(\coprod_{s \in G / U} y(U s), \mathcal{A}\right),
$$

and for any object $Z$ of $\mathcal{T}$

$$
\begin{gathered}
\operatorname{Hom}_{\mathcal{T}}\left(Z, \underline{\operatorname{Hom}}_{U}\left(\coprod_{s \in G / U} y(U s), \mathcal{A}\right)\right) \cong \operatorname{Hom}_{B U}\left(Z \times \coprod_{s \in G / U} y(U s), \mathcal{A}\right) \\
\cong \operatorname{Hom}_{B U}\left(\coprod_{s \in G / U} Z \times y(U s), \mathcal{A}\right) \cong \prod_{s \in G / U} \operatorname{Hom}_{B U}(Z \times E U, \mathcal{A}),
\end{gathered}
$$




\section{FLACH}

since coproducts in a topos are universal [SGA4, II, Exemple 4.5.1] and for any $s \in G / U$ there is an isomorphism $y(U s) \cong y(U)=E U$. The equivalence of categories of Lemma 7 (a) shows that

$$
\operatorname{Hom}_{B U}(Z \times E U, \mathcal{A}) \cong \operatorname{Hom}_{\mathcal{T}}(Z, \mathcal{A})
$$

and hence

$$
(B f){ }_{*} \mathcal{A}=\underline{\operatorname{Hom}}_{U}\left(\coprod_{s \in G / U} y(U s), \mathcal{A}\right) \cong \prod_{s \in G / U} \mathcal{A} .
$$

Since finite products are exact in the abelian category $\operatorname{Ab}(\mathcal{T})$ we find that $(B f)_{*}$ is an exact functor.

Remark. Since infinite direct products are not exact in $\operatorname{Ab}(\mathcal{T})$ it seems there is no Shapiro lemma if $U$ is open but $G / U$ is infinite. Likewise, if one just assumes that $U$ is a closed subgroup, the morphism $B f$ is in general not totally acyclic as the following lemma shows.

Lemma 8. Suppose that $f: G \rightarrow G^{\prime}$ is a closed embedding of a nontrivial discrete group into a connected group. Then $(B f)_{*}$ is not exact.

Proof. We have $(B f)_{*} \mathcal{A}=\underline{\operatorname{Hom}}_{G}\left(G^{\prime}, \mathcal{A}\right)$ and by $(5)$ the value of this sheaf on a topological space $U$ is given by

$$
\operatorname{Hom}_{B G}\left(G^{\prime} \times U, \mathcal{A}\right)=\mathcal{A}\left(G^{\prime} \times U\right)^{G} .
$$

In particular, the value at $U=\{*\}$, i.e. the stalk at the point of $\mathcal{T}$ given by the one-point topological space, is $\mathcal{A}\left(G^{\prime}\right)^{G}$. Restricting to sheaves $\mathcal{A}$ represented by discrete $G$-modules $A$ we find $\operatorname{Hom}_{\text {Top }}\left(G^{\prime}, A\right)^{G}=A^{G}$ since $G^{\prime}$ is connected. Since $G$ is nontrivial there is a short exact sequence of discrete $G$-modules the sequence of invariants of which is not exact. Hence $(B f)_{*}$ of this sequence is also not exact.

The following discussion applies to group objects in an arbitrary topos $\mathcal{T}$. Let

$$
1 \rightarrow \mathcal{H} \stackrel{i}{\rightarrow} \mathcal{G} \stackrel{p}{\rightarrow} \mathcal{Q} \rightarrow 1
$$

be an exact sequence of group objects in $\mathcal{T}$ (in the sense explained before (3)). The restriction functor $(B i)^{*}: B_{\mathcal{T}} \mathcal{G} \rightarrow B_{\mathcal{T}} \mathcal{H}$ has a left adjoint given by $(B i) !(\mathcal{X})=E \mathcal{G} \times{ }_{\mathcal{H}} \mathcal{X}$ which is the orbit space of $\mathcal{Y}:=E \mathcal{G} \times \mathcal{X}$ under its natural diagonal $\mathcal{H}$-action (right on $E \mathcal{G}$ and left on $\mathcal{X}$ ), i.e. the colimit of a diagram $\mathcal{H} \times \mathcal{Y} \rightrightarrows \mathcal{Y}$ in $\mathcal{T}$ (see also [MM92, VII.3, Theorem 1]).

LEMma 9.

(a) For any object $\mathcal{X}$ of $B_{\mathcal{T}} \mathcal{H}$ there is an equivalence of topoi

$$
B_{\mathcal{T}} \mathcal{G} /\left(E \mathcal{G} \times{ }_{\mathcal{H}} \mathcal{X}\right) \rightarrow B_{\mathcal{T}} \mathcal{H} / \mathcal{X}
$$

sending $Y \rightarrow E \mathcal{G} \times{ }_{\mathcal{H}} \mathcal{X}$ to $Y \times_{E \mathcal{G} \times \mathcal{H}} \mathcal{X}\{*\} \times \mathcal{X}$ and $Z \rightarrow \mathcal{X}$ to $E \mathcal{G} \times_{\mathcal{H}} Z \rightarrow E \mathcal{G} \times{ }_{\mathcal{H}} \mathcal{X}$.

(b) If $\mathcal{X}$ is an object of $B \mathcal{G}$ then $E \mathcal{G} \times \mathcal{H} \mathcal{X}$ with action on the first factor is isomorphic to $E \mathcal{Q} \times \mathcal{X}$ with diagonal action.

Proof. (See also [SGA4, IV.5.8].) This is an immediate generalization of Lemma 7 (which is the special case where $\mathcal{H}=1$ ).

Corollary 5. For any abelian group object $\mathcal{A}$ of $B_{\mathcal{T}} \mathcal{G}$ the object $\underline{H}^{q}(\mathcal{H}, \mathcal{A}):=R^{q} e_{\mathcal{H}, *}(B i)^{*}(\mathcal{A})$ of $\mathcal{T}\left(e_{\mathcal{H}}\right.$ defined as in (4)) carries an action of $\mathcal{Q}$ and there is a Hochschild-Serre spectral sequence

$$
H^{p}\left(\mathcal{Q}, \underline{H}^{q}(\mathcal{H}, \mathcal{A})\right) \Rightarrow H^{p+q}(\mathcal{G}, \mathcal{A}) .
$$




\section{COHOMOLOGY OF TOPOLOGICAL GROUPS}

Proof. Taking $\mathcal{X}=*$ in Lemma 9 and using (5) we have an identity of functors in the arguments $Z$ of $\mathcal{T}$ and $\mathcal{A}$ in $B_{\mathcal{T}} \mathcal{G}$, that is

$$
\begin{aligned}
& \operatorname{Hom}_{\mathcal{T}}\left(Z, e_{\mathcal{H}, *}(B i)^{*} \mathcal{A}\right)=\operatorname{Hom}_{B_{\mathcal{T}} \mathcal{H}}\left(Z,(B i)^{*} \mathcal{A}\right) \\
& \quad=\operatorname{Hom}_{B_{\mathcal{T}} \mathcal{G} / E \mathcal{Q}}\left(E \mathcal{G} \times \mathcal{H} Z, E \mathcal{G} \times_{\mathcal{H}}(B i)^{*} \mathcal{A}\right)=\operatorname{Hom}_{B_{\mathcal{T}} \mathcal{G} / E \mathcal{Q}}(E \mathcal{Q} \times Z, E \mathcal{Q} \times \mathcal{A}) \\
& =\operatorname{Hom}_{B_{\mathcal{T}} \mathcal{G}}(E \mathcal{Q} \times Z, \mathcal{A})=\operatorname{Hom}_{\mathcal{T}}\left(Z, \underline{\operatorname{Hom}}_{\mathcal{G}}(E \mathcal{Q}, \mathcal{A})\right)=\operatorname{Hom}_{\mathcal{T}}\left(Z,(B p)_{*} \mathcal{A}\right)
\end{aligned}
$$

where the last identity is [MM92, VII.3(12)] (note that this reference has a misprint: $H$ should be $G)$. Hence we have an identity of functors $e_{\mathcal{H}, *}(B i)^{*}=(B p)_{*}$ on $\operatorname{Ab}\left(B_{\mathcal{T}} \mathcal{G}\right)$. By [MM92, VII.3, Theorem 1] the functor $B i^{*}$ has both a left and a right adjoint, hence is exact. By Lemma 9(a) $B_{\mathcal{T}} \mathcal{H}$ is a slice topos over $B_{\mathcal{T}} \mathcal{G}$ and hence the functor $(B i)_{!}: \operatorname{Ab}\left(B_{\mathcal{T}} \mathcal{H}\right) \rightarrow \operatorname{Ab}\left(B_{\mathcal{T}} \mathcal{G}\right)$ is exact and the functor $(B i)^{*}$ maps injective objects in $\operatorname{Ab}\left(B_{\mathcal{T}} \mathcal{G}\right)$ to injective objects in $\operatorname{Ab}\left(B_{\mathcal{T}} \mathcal{H}\right)$. Therefore $R^{q}(B p)_{*}=R^{q} e_{\mathcal{H}, *}(B i)^{*}=\underline{H}^{q}(\mathcal{H},-)$ and the Hochschild-Serre spectral sequence coincides with the Leray spectral sequence for the morphism $B p$.

Corollary 6. Let

$$
1 \rightarrow H \stackrel{i}{\rightarrow} G \stackrel{p}{\rightarrow} Q \rightarrow 1
$$

be an exact sequence of topological groups so that $p$ is a local section cover and let $A$ be a topological $G$-module. Then there is a Hochschild-Serre spectral sequence

$$
H^{p}\left(Q, \underline{H}^{q}(H, A)\right) \Rightarrow H^{p+q}(G, A),
$$

where however the objects $\underline{H}^{q}(H, A)$ of $\mathcal{T}=\operatorname{Sh}\left(\right.$ Top, $\left.J_{\text {open }}\right)$ are not in general representable by topological Q-modules.

Proof. This is immediate from Lemma 4.

Proposition 6.2. If $\mathcal{G}$ and $\mathcal{G}^{\prime}$ are group objects in a topos $\mathcal{T}$ then the natural geometric morphism

$$
B_{\mathcal{T}}\left(\mathcal{G} \times \mathcal{G}^{\prime}\right) \rightarrow B_{\mathcal{T}} \mathcal{G} \times \mathcal{T} B_{\mathcal{T}} \mathcal{G}^{\prime}
$$

is an equivalence of topoi. In particular, for topological groups $G$ and $G^{\prime}$ we have

$$
B\left(G \times G^{\prime}\right) \cong B G \times_{\mathcal{T}} B G^{\prime},
$$

where $\mathcal{T}=\operatorname{Sh}\left(\right.$ Top, $\left.J_{\text {open }}\right)$.

Proof. This is [Dia75, Theorem 5.1] using the fact that $B_{B_{\mathcal{T}} \mathcal{G}}\left(\Delta\left(\mathcal{G}^{\prime}\right)\right)=B_{\mathcal{T}}\left(\mathcal{G} \times \mathcal{G}^{\prime}\right)$ where $\Delta: \mathcal{T} \rightarrow$ $B_{\mathcal{T}} \mathcal{G}$ sends an object to itself with trivial $\mathcal{G}$-action (this is the pullback functor for the geometric morphism $B_{\mathcal{T}} \mathcal{G} \rightarrow \mathcal{T}$ ). In fact both categories coincide with the category of objects in $\mathcal{T}$ equipped with commuting actions of $\mathcal{G}$ and $\mathcal{G}^{\prime}$. We also refer to [Dia75] for a general discussion of fibered products of topoi.

Unfortunately we were not able to deduce from this proposition a general Kuenneth formula for topological group cohomology. Below we shall prove a Kuenneth formula for compact groups and $\mathbb{Z}$-coefficients.

\section{The small classifying topos of a topological group}

For any topological group $G$ there is another classifying topos $B^{s m} G$ considered in Moerdijk's book [Moe95] and also in [MM92, III.9]. $B^{s m} G$ is the full subcategory of $B_{\text {Top }} G$ consisting of discrete sets $X$ equipped with a continuous $G$-action. Hence we have a fully faithful functor

$$
p^{*}: B^{s m} G \rightarrow B_{\text {Top }} G \stackrel{i}{\rightarrow} B G .
$$




\section{FLACH}

Proposition 7.1. The functor $p^{*}$ is the pullback functor for a (connected) morphism of topoi $p$ : $B G \rightarrow B^{s m} G$ where $p_{*} \mathcal{F}$ is given by the discrete set $\mathcal{F}(\{*\})^{\delta}$ with its action of $\operatorname{Hom}_{\text {Top }}(\{*\}, G)=G$. Here, for any $G$-set $X$, we denote by $X^{\delta}=\bigcup_{U \subseteq G \text { open }} X^{U} \subseteq X$ the maximal subset on which the $G$-action is continuous.

Proof. We consider the inclusion $j: B^{s m} G \rightarrow B_{\text {Top }} G$ as a functor between sites where $B^{s m} G$ has the canonical topology and $B_{\text {Top }} G$ the local section topology. Then $j$ preserves finite limits (in both categories these are computed on the underlying sets with the induced topology and $G$-action) as well as covers (since a surjection of discrete spaces is a local section cover), so it is a morphism of sites [SGA4, IV.4.9]. By [SGA4, Proposition IV.4.9.4] and Corollary 2 the functor $j$ induces a morphism of topoi

$$
p: B G=\operatorname{Sh}\left(B_{\text {Top }} G, J_{l s}\right) \rightarrow \operatorname{Sh}\left(B^{s m} G, J_{\text {can }}\right)=B^{s m} G .
$$

If $X$ is a discrete $G$-space an element of $\operatorname{Hom}_{B G}\left(p^{*} X, \mathcal{F}\right)$ is an element $\xi$ of

$$
\operatorname{Hom}_{\mathcal{T}}(y X, \mathcal{F})=\mathcal{F}(X)=\operatorname{Hom}_{\text {Set }}(X, \mathcal{F}(\{*\}))
$$

which commutes with the $y G$-action. This is the case if for all $\psi \in \operatorname{Hom}_{\text {Set }}(X, G)$ and $x \in X$ one has $\psi(x) \xi(x)=\xi(\psi(x) x)$, i.e. if for all $g \in G$ and $x \in X$ one has $g \xi(x)=\xi(g x)$. This is equivalent to $\xi \in \operatorname{Hom}_{B} \operatorname{Set}_{G}(X, \mathcal{F}(\{*\}))=\operatorname{Hom}_{B^{s m} G}\left(X, \mathcal{F}(\{*\})^{\delta}\right)$.

Finally, by definition, $p$ is called connected if $p^{*}$ is fully faithful.

We shall see in $\S 9$ that $p$ is acyclic if $G$ is totally disconnected, locally compact and paracompact. On the other hand, if $G$ is connected then the action of $G$ on any discrete set is trivial and $p$ coincides with the canonical morphism $B G \rightarrow$ Set giving rise to group cohomology.

\section{Compact groups and continuity}

The following proposition generalizes a well known fact from the cohomology theory of profinite groups. If $G_{\alpha}$ is an inverse system of topological groups, a direct system of objects of $B G_{\alpha}$ is a family $\mathcal{A}_{\alpha}$ together with morphisms $(B g)^{*} \mathcal{A}_{\alpha} \rightarrow \mathcal{A}_{\beta}$ in $B G_{\beta}$ for each $G_{\beta} \stackrel{g}{\rightarrow} G_{\alpha}$ so that $(B h)^{*}(B g)^{*} \mathcal{A}_{\alpha} \rightarrow$ $(B h)^{*} \mathcal{A}_{\beta} \rightarrow \mathcal{A}_{\gamma}$ equals $(B g \circ B h)^{*}: \mathcal{A}_{\alpha} \rightarrow \mathcal{A}_{\gamma}$ for any composite $G_{\gamma} \stackrel{h}{\rightarrow} G_{\beta} \stackrel{g}{\rightarrow} G_{\alpha}$.

Proposition 8.1. Let $G=\varliminf_{\alpha} G_{\alpha}$ be a filtered inverse limit of compact groups and $\mathcal{A}_{\alpha}$ a direct system of objects of $B G_{\alpha}$. Denote by $p_{\alpha}: G \rightarrow G_{\alpha}$ the natural projection and put $\mathcal{A}:=\lim _{\longrightarrow}\left(B p_{\alpha}\right)^{*} \mathcal{A}_{\alpha}$. Assume that for all $n \geqslant 1$ the natural morphism of sheaves on $G^{n}$ (see Lemma 2)

$$
\underset{\alpha}{\lim _{\alpha}} p_{\alpha, *}^{n, \mathcal{A}_{\alpha} G_{\alpha}^{n}} \rightarrow \underset{\alpha}{\lim _{\alpha}} \mathcal{A}_{\alpha, G^{n}}=\mathcal{A}_{G^{n}}
$$

is an isomorphism. Then

$$
H^{i}(G, \mathcal{A})=\underset{\alpha}{\lim } H^{i}\left(G_{\alpha}, \mathcal{A}_{\alpha}\right) .
$$

Proof. The Cartan-Leray spectral sequence (7) is contravariantly functorial in $G$ and covariantly functorial in $\mathcal{A}$. Since a direct limit over a filtered index category (in the category of abelian groups) is exact we obtain a map of spectral sequences

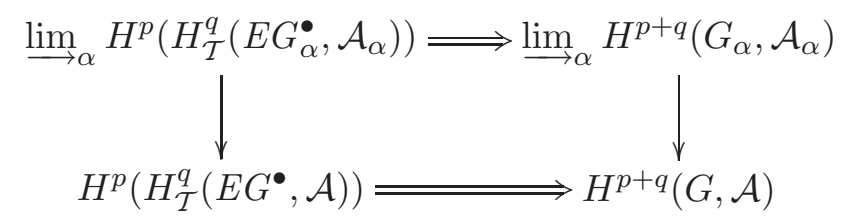




\section{COHOMOLOGY OF TOPOLOGICAL GROUPS}

and it suffices to show that the map on the initial terms is an isomorphism. Again using exactness of the direct limit we have

$$
\begin{aligned}
& \underset{\alpha}{\lim } H^{p}\left(H_{\mathcal{T}}^{q}\left(E G_{\alpha}^{\bullet}, \mathcal{A}_{\alpha}\right)\right)=H^{p}\left(\underset{\alpha}{\lim } H_{\mathcal{T}}^{q}\left(E G_{\alpha}^{\bullet}, \mathcal{A}_{\alpha}\right)\right)=H^{p}\left(\underset{\alpha}{\lim } H^{q}\left(G_{\alpha}^{\bullet}, \mathcal{A}_{\alpha, G_{\alpha}^{\bullet}}\right)\right) \\
& \quad=H^{p}\left(H^{q}(\underbrace{\lim }_{\alpha} G_{\alpha}^{\bullet}, \underset{\alpha}{\lim } p_{\alpha}^{*} \mathcal{A}_{\alpha, G_{\alpha}^{\bullet}})\right)=H^{p}\left(H^{q}\left(G^{\bullet}, \mathcal{A}_{G^{\bullet}}\right)\right)=H^{p}\left(H_{\mathcal{T}}^{q}\left(E G^{\bullet}, \mathcal{A}\right)\right),
\end{aligned}
$$

where the third equality follows from a standard continuity theorem in the sheaf cohomology of compact Hausdorff spaces [Bre97, Theorem 14.4], applied to the spaces $G^{n}$, and the fourth equality is our assumption (9) on the direct system $\mathcal{A}_{\alpha}$.

Corollary 7. Assume that in the situation of Proposition 8.1 the sheaves $\mathcal{A}_{\alpha}$ are representable by discrete continuous $G_{\alpha}$-modules $A_{\alpha}$. Then (9) is satisfied and

$$
H^{i}\left(G, \underset{\alpha}{\lim } A_{\alpha}\right)=\underset{\alpha}{\lim } H^{i}\left(G_{\alpha}, A_{\alpha}\right) .
$$

Proof. For any continuous map $f: X \rightarrow Y$ of topological spaces and discrete abelian group $A$ we have $f^{*} A_{Y}=A_{X}$ where $A_{X}$ is the constant sheaf represented by $A$. Hence $p_{\alpha}^{n, *} A_{\alpha, G_{\alpha}^{n}}=A_{\alpha, G^{n}}$ and (9) holds by passing to the limit.

Proposition 8.2. If $G_{1}$ and $G_{2}$ are compact groups then

$$
R \Gamma\left(G_{1} \times G_{2}, \mathbb{Z}\right) \cong R \Gamma\left(G_{1}, \mathbb{Z}\right) \bigotimes_{\mathbb{Z}}^{L} R \Gamma\left(G_{2}, \mathbb{Z}\right)
$$

in the derived category of abelian groups.

Proof. Each compact group is a filtered inverse limit of compact Lie groups [Wei51, § 25] so we may write $G_{i}=\lim _{\longleftarrow} G_{i, \alpha}$ where the $G_{i, \alpha}$ are compact Lie groups. We have

$$
\begin{aligned}
& H^{n}\left(G_{1} \times G_{2}, \mathbb{Z}\right) \cong \underset{(\overrightarrow{\alpha, \beta})}{\lim _{(}} H^{n}\left(G_{1, \alpha} \times G_{2, \beta}, \mathbb{Z}\right) \\
& \cong \underset{(\overrightarrow{\alpha, \beta})}{\lim } H_{\text {sing }}^{n}\left(\mathbf{B}\left(G_{1, \alpha} \times G_{2, \beta}\right), \mathbb{Z}\right) \\
& \cong \underset{(\alpha, \beta)}{\lim } H_{\text {sing }}^{n}\left(\mathbf{B}\left(G_{1, \alpha}\right) \times \mathbf{B}\left(G_{2, \beta}\right), \mathbb{Z}\right),
\end{aligned}
$$

where the first (respectively second) equality follows from Corollary 7 (respectively Proposition 5.2) and the third follows from

$$
\mathbf{B}\left(G_{1} \times G_{2}\right) \cong \mathbf{B} G_{1} \times \mathbf{B} G_{2}
$$

for arbitrary compactly generated topological groups when the product on the right is taken in the category of compactly generated spaces.

For a compact Lie group $G$ the abelian groups $H^{i}(G, \mathbb{Z})=H^{i}(\mathbf{B}(G), \mathbb{Z})$ are finitely generated as can be seen from the spectral sequence $(7)$ and the fact that the cohomology groups $H_{\mathcal{T}}^{q}\left(G^{n}, \mathbb{Z}\right)=$ $H_{\text {sing }}^{q}\left(G^{n}, \mathbb{Z}\right)$ of the compact manifolds $G^{n}$ are finitely generated. Hence there is a short exact Kuenneth sequence

$$
\begin{aligned}
0 & \rightarrow \bigoplus_{p+q=n} H_{\text {sing }}^{p}\left(\mathbf{B}\left(G_{1, \alpha}\right), \mathbb{Z}\right) \otimes_{\mathbb{Z}} H_{\text {sing }}^{q}\left(\mathbf{B}\left(G_{2, \beta}\right), \mathbb{Z}\right) \rightarrow H_{\text {sing }}^{n}\left(\mathbf{B}\left(G_{1, \alpha}\right) \times \mathbf{B}\left(G_{2, \beta}\right), \mathbb{Z}\right) \\
& \rightarrow \bigoplus_{p+q=n+1} \operatorname{Tor}_{1}^{\mathbb{Z}}\left(H_{\text {sing }}^{p}\left(\mathbf{B}\left(G_{1, \alpha}\right), \mathbb{Z}\right), H_{\text {sing }}^{q}\left(\mathbf{B}\left(G_{2, \beta}\right), \mathbb{Z}\right)\right) \rightarrow 0 .
\end{aligned}
$$




\section{FLACH}

Applying the filtered direct limit $\lim _{(\alpha, \beta)}$ to this sequence and using the fact that $-\otimes_{\mathbb{Z}}-$ and $\operatorname{Tor}_{1}^{\mathbb{Z}}(-,-)$ commute with direct limits we obtain an exact sequence

$$
\begin{aligned}
0 & \rightarrow \bigoplus_{p+q=n} H^{p}\left(G_{1}, \mathbb{Z}\right) \otimes_{\mathbb{Z}} H^{q}\left(G_{2}, \mathbb{Z}\right) \rightarrow H^{n}\left(G_{1} \times G_{2}, \mathbb{Z}\right) \\
& \rightarrow \bigoplus_{p+q=n+1} \operatorname{Tor}_{1}^{\mathbb{Z}}\left(H^{p}\left(G_{1}, \mathbb{Z}\right), H^{q}\left(G_{2}, \mathbb{Z}\right)\right) \rightarrow 0,
\end{aligned}
$$

which shows that (11) is a quasi-isomorphism.

\section{Locally compact groups}

In this section we discuss the groups $H^{i}(G, \mathcal{A})$ under the assumption that $G$ is locally compact. In fact all the results below show that $H^{i}(G, \mathcal{A})$ agrees with continuous cochain cohomology under various assumptions. We first record the following general result which shows that one can replace Top by a suitable full subcategory without changing the groups $H^{i}(G, \mathcal{A})$.

Proposition 9.1. Let Top' be a full subcategory of Top closed under finite limits and so that any open subspace of a space in Top' again lies in $\mathrm{Top}^{\prime}$. Set $\mathcal{T}^{\prime}=\mathrm{Sh}\left(\mathrm{Top}^{\prime}, J_{\text {open }}\right)$ and for any group object $G$ of Top' set $B^{\prime} G=B_{\mathcal{T}^{\prime}}\left(y^{\prime}(G)\right)$. Then there is a commutative diagram of geometric morphisms

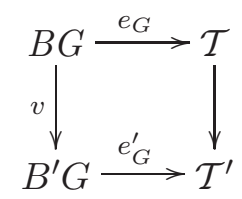

where the vertical arrows are totally acyclic homotopy equivalences. In particular for any abelian group object $\mathcal{A}$ of $B G$ we have $H^{i}(G, \mathcal{A})=H^{i}\left(B^{\prime} G, v_{*} \mathcal{A}\right)$.

Proof. The inclusion functor Top' $\rightarrow$ Top commutes with finite limits, preserves covers [MM92, p. 411] and has the covering lifting property [MM92, p. 412] for the open covering topology on both categories. The discussion on [MM92, p. 416] then shows that there is a totally acyclic homotopy equivalence $\mathcal{T} \rightarrow \mathcal{T}^{\prime}$. The same applies to the functor $G \times$ Top $^{\prime} \rightarrow G \times$ Top, where the notation is as in Corollary 3, and hence there is a totally acyclic homotopy equivalence $v: B G \rightarrow B^{\prime} G$ extending $\mathcal{T} \rightarrow \mathcal{T}^{\prime}$.

By [Bou89, I, §9.7, Propositions 13 and 14] the category Top ${ }^{l c}$ of locally compact spaces with countable basis satisfies the assumptions of Proposition 9.1. We denote $\mathcal{T}^{\prime}$ (respectively $B^{\prime} G$, respectively $e_{G}^{\prime}$ ) by $\mathcal{T}^{l c}$ (respectively $B^{l c} G$, respectively $e_{G}^{l c}$ ) in this case. The following proposition highlights one advantage of working with $\mathcal{T}^{l c}$ in that the generalized topology on the groups $H^{i}(G, \mathcal{A})$ can sometimes be shown to be an actual topology.

Proposition 9.2. If $G$ is locally compact with countable basis then $H^{i}(G, \mathcal{A}) \cong H^{i}\left(B^{l c} G, v_{*} \mathcal{A}\right)$. Moreover, if $\mathcal{A}$ is represented by a discrete $G$-module $A$, then the generalized topology on $H^{i}\left(B^{l c} G, A\right)$ is the discrete topology, i.e. the object $R^{i} e_{G, *}^{l c} A$ of $\mathcal{T}^{l c}$ is representable by the discrete group $H^{i}\left(B^{l c} G, A\right)$.

Proof. The first statement is Proposition 9.1. For any object $\mathcal{F}$ of $\mathcal{T}^{l c}$ and locally compact topological space $q: X \rightarrow\{*\}$ we have a morphism $q^{*} \mathcal{F}(\{*\}) \rightarrow \mathcal{F}_{X}$ of sheaves (functors on open subsets) on $X$ and $\mathcal{F}$ is represented by the discrete group $\mathcal{F}(\{*\})$ if and only if this morphism is an isomorphism for all $X$. This in turn can be checked on stalks. For any abelian group object $\mathcal{A}$ of $B^{l c} G$ the sheaf $\mathcal{F}=R^{i} e_{G, *}^{l c} \mathcal{A}$ is the associated sheaf of the presheaf $\tilde{\mathcal{F}}(U):=H_{B^{l c} G}^{i}\left(e_{G}^{l c, *} U, \mathcal{A}\right)$ on 


\section{COHOMOLOGY OF TOPOLOGICAL GROUPS}

Top $^{l c}$ and its stalk at $x \in X$ is therefore given by

$$
\left(R^{i} e_{G, *}^{l c} \mathcal{A}\right)_{x}=\lim _{x \in U \subseteq X} H_{B^{l c} G}^{i}\left(e_{G}^{l c, *} U, \mathcal{A}\right)
$$

For any topological space $U$, the projection $G \times U \rightarrow U$ is a local section cover, hence gives rise to an epimorphism $E G \times U \rightarrow U$ in $B^{l c} G$ which in turn gives rise to the simplicial object

$$
\cdots \rightarrow E G \times E G \times E G \times U \rightarrow E G \times E G \times U \rightarrow E G \times U
$$

and the corresponding Cartan-Leray spectral sequence

$$
H^{p}\left(H_{\mathcal{T}^{l c}}^{q}\left(E G^{\bullet} \times U, \mathcal{A}\right)\right) \Rightarrow H_{B^{l c} G}^{p+q}\left(e_{G}^{l c, *} U, \mathcal{A}\right),
$$

functorial in $U$. The argument here is identical to the proof of the spectral sequence in (7), using Lemma 7 for $\mathcal{X}=y U$ instead of $\mathcal{X}=y\{*\}$. Since the colimit in (13) is filtered, hence exact, we obtain a spectral sequence

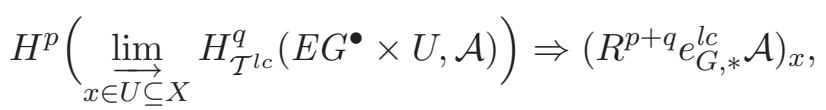

functorial in the pair $(X, x)$. Indeed, given a continuous map $f:(X, x) \rightarrow(Y, y)$ of pointed spaces, and induced map $f:(U, x) \rightarrow(V, y)$ of open neighborhoods, we obtain the following map of spectral sequences.

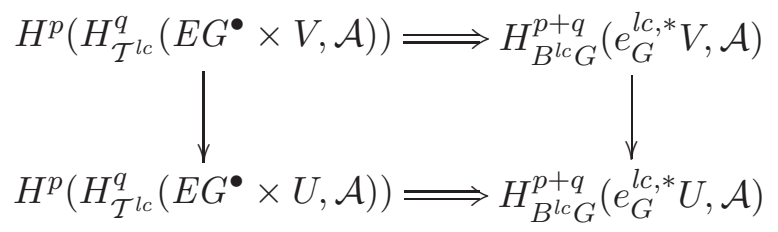

Taking the limit (first over neighborhoods of $x$, then over neighborhoods of $y$ ) we then obtain the following map of spectral sequences.

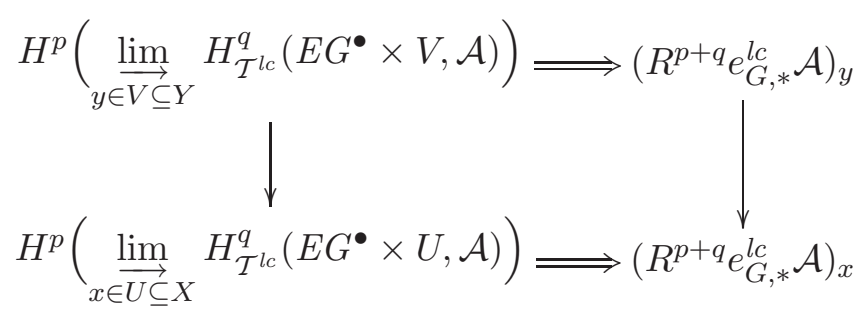

Taking $f$ to be $q:(X, x) \rightarrow(\{*\}, *)$ we obtain a map of spectral sequences

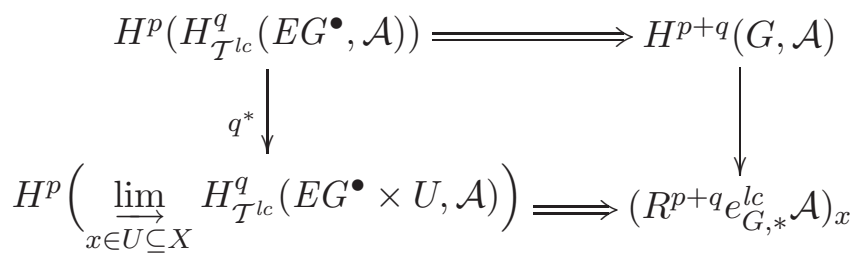

since all neighborhoods $* \in V$ reduce to $\{*\}$. We now assume that $\mathcal{A}=y A$ is represented by a discrete $G$-module $A$. By [Bre97, Theorem 10.6] (with $\Phi$ the family of all closed sets which is paracompactifying) the natural restriction map

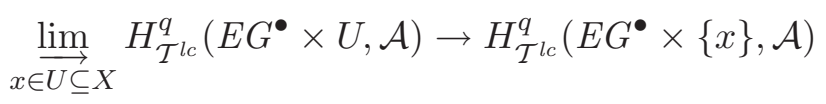

is an isomorphism, since for any $n$ the restriction of $\mathcal{A}_{E G^{n} \times X}$, the constant sheaf represented by $A$, to the closed subspace $E G^{n} \times\{x\}$ of $E G^{n} \times X$ is again the constant sheaf represented by $A$, 


\section{FLACH}

i.e. coincides with $\mathcal{A}_{E G^{n} \times\{x\}}$. Since the restriction map splits the map $q^{*}$, the map of spectral sequences (14) is an isomorphism on initial terms and therefore an isomorphism on end terms. We conclude that the morphism of sheaves $q^{*} H^{i}(G, A) \rightarrow\left(R^{i} e_{G, *}^{l c} A\right)_{X}$ is an isomorphism.

Remark. The category of locally compact spaces also satisfies Proposition 9.1 and the first statement of Proposition 9.2 has an analog for any locally compact group $G$. However, to compute stalks as in the proof of Proposition 9.2 we have to assure that all spaces are paracompact. For a locally compact space as well as all its open subspaces this is assured by assuming a countable basis.

\subsection{Discrete groups}

If $G$ is discrete then we may take Top $^{\prime}=$ Set, the category of discrete topological spaces, in Proposition 9.1 and $B^{\prime} G=B_{\text {Set }} G$ is then simply the category of $G$-sets. Proposition 9.1 implies the following result.

Proposition 9.3. (See also [Lic04, Lemma 5.1].) If $G$ is discrete then $H^{i}(G, \mathcal{A})$ agrees with the usual group cohomology of the $G$-module $\mathcal{A}(\{*\})$. In particular, if $G=\mathbb{Z}$ then $H^{i}(G, \mathcal{A})$ is concentrated in degrees zero and one.

\subsection{Totally disconnected groups}

This includes profinite groups and groups of rational points of algebraic groups over local fields. If $G$ is profinite and $A$ a discrete continuous $G$-module then $H^{i}(G, A)$ is usually defined as right derived functor of the invariant functor $A \mapsto A^{G}$, i.e. as cohomology in the topos $B^{s m} G$. If $A$ is just a continuous $G$-module then $H^{i}(G, A)$ is usually defined via continuous cochains and it is shown that both definitions agree for discrete $A$. The following proposition shows that both definitions also agree with $H^{i}(G, A)$ as defined in this paper.

Proposition 9.4. If $G$ is a totally disconnected, locally compact paracompact group then $p: B G \rightarrow$ $B^{s m} G$ is acyclic. In particular $H^{i}\left(G, p^{*} A\right)=H^{i}\left(B^{s m} G, A\right)$ for any discrete continuous $G$-module $A$. Moreover, $H^{i}(G, \mathcal{A})$ is computed by the complex in Proposition 5.1 for any abelian group object $\mathcal{A}$ of $B G$.

Proof. All the spaces $G^{n}$ are again totally disconnected, locally compact and paracompact. Since such a space has vanishing sheaf cohomology in positive degrees (any open cover has a refinement by a disjoint cover) Proposition 5.1 applies and gives the last statement.

Recall from [MM92, III.9, Theorem 1] that $B^{s m} G=\operatorname{Sh}\left(S(G), J_{\text {atomic }}\right)$ where $S(G)$ is the full subcategory of objects of $B^{s m} G$ of the form $G / U$ with $U$ an open subgroup. The sheaf $R^{i} p_{*} p^{*} A$ is the sheaf associated to the presheaf $G / U \mapsto H^{i}\left(p^{*}(G / U), p^{*} A\right)=H^{i}\left(U, p^{*} A\right)$. The last identity follows since $G \rightarrow G / U$ has a continuous section, hence by Lemma 4 the effective equivalence relation given by $U$ on $G$ is mapped by $y$ to an effective equivalence relation in $\mathcal{T}$ with quotient $y(G / U)$. Then by Lemma 9 we have an equivalence of topoi $B G /(y(G / U)) \rightarrow B U$. Now since $U$ is again totally disconnected $H^{i}\left(U, p^{*} A\right)$ is computed by continuous cochains. Since $A$ is discrete, each such cochain $\phi$ is constant on a smaller open subgroup $U^{\prime} \subseteq U$ and hence the image of $\phi$ vanishes in $H^{i}\left(U^{\prime}, p^{*} A\right)=H^{i}\left(p^{*}\left(G / U^{\prime}\right), p^{*} A\right)$ for $i>0$. Since $G / U^{\prime} \rightarrow G / U$ is a covering for the atomic topology, the sheaf $R^{i} p_{*} p^{*} A$ vanishes.

We note that $p$ is in general not totally acyclic. For example, if $G=\mathbb{Z}_{p}$ and $A=\mathbb{Z}_{p}$ with trivial $G$-action and its profinite topology, then $R^{i} p_{*}(y A)=0$ for $i \neq 0,1$ and $p_{*}(y A)=\mathbb{Z}_{p}^{\delta}$ (discrete topology), $R^{1} p_{*}(y A)=\mathbb{Q}_{p}^{\delta}$. The Leray spectral sequence for $p$ degenerates to an isomorphism $\mathbb{Z}_{p}=H^{0}\left(B^{s m} G, \mathbb{Z}_{p}^{\delta}\right)=H^{0}\left(G, \mathbb{Z}_{p}\right)$ and an exact sequence

$$
0 \rightarrow H^{1}\left(G, \mathbb{Z}_{p}\right) \rightarrow H^{0}\left(B^{s m} G, R^{1} p_{*} \mathbb{Z}_{p}\right)=\mathbb{Q}_{p}^{\delta} \rightarrow H^{2}\left(B^{s m} G, \mathbb{Z}_{p}^{\delta}\right)=\mathbb{Q}_{p} / \mathbb{Z}_{p} \rightarrow 0,
$$

since $H^{2}\left(B^{s m} G, \mathbb{Z}_{p}^{\delta}\right) \cong H^{1}\left(B^{s m} G, \mathbb{Q}_{p} / \mathbb{Z}_{p}\right)=\mathbb{Q}_{p} / \mathbb{Z}_{p}$. 


\section{COHOMOLOGY OF TOPOLOGICAL GROUPS}

\subsection{Vector space coefficients}

We denote by $\tilde{\mathbb{R}}:=y(\mathbb{R})$ the ring object of $\mathcal{T}$ represented by $\mathbb{R}$ with its standard topology.

Proposition 9.5. Suppose $G$ is locally compact and paracompact. If $\mathcal{A}$ is a sheaf of $\tilde{\mathbb{R}}$-modules then $H^{i}(G, \mathcal{A})$ agrees with the cohomology of the complex in Proposition 5.1. In particular if $V$ is a topological $\mathbb{R}$-vector space with a continuous $G$-action then $H^{i}(G, V)$ is computed by continuous cochains.

Proof. If $G$ is locally compact and paracompact, so are all the spaces $G^{n}$ [Bou89, I, $\S 9.9$ ]. Since a sheaf of $\mathbb{R}$-modules on a paracompact space is soft [Bre97, Theorem 9.16] it has no cohomology in positive degrees [Bre97, Theorem 9.11]. We conclude by Proposition 5.1.

This proposition makes available all the results of the book [BW00, ch. IX] by Borel and Wallach concerning continuous cochain cohomology of locally compact groups with vector space coefficients. For example one has the following result.

Corollary 8. Suppose $V$ is a locally convex, Hausdorff, quasi-complete topological vector space over $\mathbb{R}$ with a continuous action of the compact group $G$. Then $H^{i}(G, V)=0$ for $i>0$.

Proof. This is [BW00, IX, Proposition 1.12].

Proposition 9.6. If $A$ is a discrete abelian group then $H^{i}(\mathbb{R}, A)=0$ for $i>0$. If $V$ is a locally convex, Hausdorff, quasi-complete topological $\mathbb{R}$-vector space with a $\mathbb{R}$-linear differentiable action of $\mathbb{R}$, then $H^{i}(\mathbb{R}, V)=0$ for $i>1$.

Proof. If $A$ is discrete then Proposition 5.1 shows that $H^{i}(\mathbb{R}, A)$ is the cohomology of the continuous cochain complex because the space $\mathbb{R}^{n}$ has no higher cohomology with constant coefficients $A$. On the other hand, any continuous map from $\mathbb{R}^{n}$ to $A$ is constant since $\mathbb{R}^{n}$ is connected, so the continuous cochain complex is the complex associated to the constant simplicial object $A$, hence acyclic in degrees $i>0$.

If $V$ is as in the lemma then $H^{i}(\mathbb{R}, V)$ agrees with the cohomology of the Lie algebra of $\mathbb{R}$ by [BW00, IX, Lemma 5.2, Proposition 5.6], hence vanishes in degrees $i>1$.

Unfortunately we were not able to compute the groups $H^{i}(\mathbb{R}, \mathcal{A})$ for arbitrary coefficients $\mathcal{A}$, even if the action of $\mathbb{R}$ is trivial. Does one always have $H^{i}(\mathbb{R}, \mathcal{A})=0$ for $i>1$ ?

\section{Cohomology of the Weil group}

For any number field $F$ and any set $S$ of places of $F$ containing the archimedean places we denote by $C_{F, S}=\mathbb{A}_{F}^{\times} / F^{\times} \cdot U_{F, S}$ the $S$-idele class group of $F$. Here $U_{F, S} \subseteq \mathbb{A}_{F}^{\times}$is the subgroup of ideles with component 1 (respectively component in $\mathcal{O}_{F_{v}}^{\times}$) at places $v \in S$ (respectively $v \notin S$ ). We allow $S$ to be infinite; if $S$ is the set $S(F)$ of all places then $C_{F}:=C_{F, S(F)}=\mathbb{A}_{F}^{\times} / F^{\times}$is the idele class group.

The Weil group $W_{F}$ of $F$ (or a Weil group of $F$ ) is a topological group defined as the projective limit

$$
W_{F}:=\lim _{K / F, S} W_{K / F, S}
$$

over either all finite Galois extensions $K / F$ and $S=S(K)$, or all finite Galois extensions $K / F$ and finite sets $S$ of places of $K$ containing the archimedean ones and the places ramified in $K / F$ (the equivalence of the two definitions is [Lic04, Lemma 3.1]). Here $W_{K / F, S}$ is the group extension

$$
1 \rightarrow C_{K, S} \rightarrow W_{K / F, S} \rightarrow \operatorname{Gal}(K / F) \rightarrow 1
$$




\section{FLACH}

corresponding to the canonical class in $H^{2}\left(\operatorname{Gal}(K / F), C_{K, S}\right)$. There is a short exact sequence of inverse systems

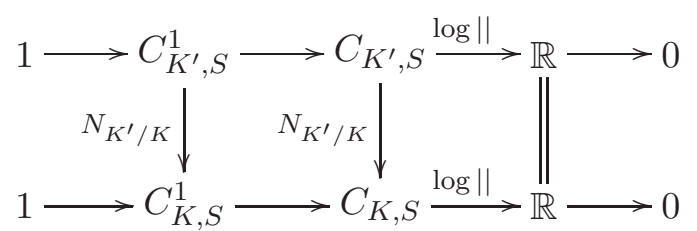

where $C_{K, S}^{1}$ is a compact Hausdorff group. Since the inclusion $C_{K, S}^{1} \rightarrow C_{K, S}$ induces an isomorphism on $H^{2}(\operatorname{Gal}(K / F),-)$ the canonical class also leads to compact Hausdorff groups $W_{F / K, S}^{1}$ and $W_{F}^{1}$. There are direct product decompositions

$$
W_{F} \cong W_{F}^{1} \times \mathbb{R}, \quad W_{K / F, S} \cong W_{K / F, S}^{1} \times \mathbb{R}
$$

in the category of topological groups.

Lemma 10. Let $A$ be either a discrete, continuous $W_{F}$-module or $A=\mathbb{R}$ with trivial $W_{F}$-action. Then

$$
H^{i}\left(W_{F}, A\right)=\underset{K / F, S}{\lim _{K, S}} H^{i}\left(W_{K / F, S}, A^{W_{K / F, S}}\right),
$$

i.e. the definition of $H^{i}\left(W_{F}, A\right)$ as a direct limit in [Lic04, Definition 3.2] coincides with topological group cohomology. If $A$ is discrete we have $H^{i}\left(W_{F}, A\right)=H^{i}\left(W_{F}^{1}, A\right)$ and if $A=\mathbb{R}$ we have $H^{i}\left(W_{F}, \mathbb{R}\right)=H^{i}(\mathbb{R}, \mathbb{R})$.

Proof. If $A$ is discrete then

$$
A=\underline{\lim } A^{W_{K / F, S}}
$$

and since the limit in (15) is filtered Corollary 7 applies to the compact group $W_{F}^{1}$ to give

$$
H^{i}\left(W_{F}^{1}, A\right)=\underset{K / F, S}{\lim _{K}} H^{i}\left(W_{K / F, S}^{1}, A^{W_{K / F, S}}\right) .
$$

The Hochschild--Serre spectral sequence

$$
H^{p}\left(G^{1}, \underline{H}^{q}(\mathbb{R}, A)\right) \Rightarrow H^{p+q}(G, A)
$$

associated to the group extension $0 \rightarrow \mathbb{R} \rightarrow G \rightarrow G^{1} \rightarrow 1$ for both $G=W_{F}$ and $G=W_{K / F, S}$ degenerates by Propositions 9.2 and 9.6, and gives isomorphisms $H^{i}\left(W_{F}, A\right) \cong H^{i}\left(W_{F}^{1}, A\right)$ and $H^{i}\left(W_{K / F, S}, A\right) \cong H^{i}\left(W_{K / F, S}^{1}, A\right)$ (see also [Lic04, §3] for this argument).

If $A=\mathbb{R}$ with its standard topology it is not true that

$$
p_{K, S}^{*} \operatorname{Hom}_{\mathrm{Top}}(-, \mathbb{R})_{W_{K / F, S}}=\operatorname{Hom}_{\mathrm{Top}}(-, \mathbb{R})_{W_{F}},
$$

where $p_{K, S}: W_{F} \rightarrow W_{K / F, S}$ is the natural continuous map, and neither is condition (9) satisfied. However, we can follow the computations in [Lic04, Lemma 3.4] and use the Hochschild-Serre spectral sequence of [BW00, ch. IX, Theorem 4.3]

$$
H^{p}\left(\mathbb{R}, H^{q}\left(G^{1}, \mathbb{R}\right)\right) \Rightarrow H^{p+q}(G, \mathbb{R})
$$

associated to the group extension $1 \rightarrow G^{1} \rightarrow G \rightarrow \mathbb{R} \rightarrow 0$ for both $G=W_{F}$ and $G=W_{K / F, S}$ together with Corollary 8 to deduce

$$
H^{i}\left(W_{F}, \mathbb{R}\right) \cong H^{i}(\mathbb{R}, \mathbb{R}) \cong H^{i}\left(W_{K / F, S}, \mathbb{R}\right)=\underset{K / F, S}{\lim _{K}} H^{i}\left(W_{K / F, S}, \mathbb{R}\right) .
$$

The following result is proved in [Lic04, Theorem 3.6] for $i \leqslant 3$ and any number field $F$. We restrict to totally imaginary $F$ to ease notation in the proof although the method of proof will quite likely extend to general $F$. 


\section{COHOMOLOGY OF TOPOLOGICAL GROUPS}

Theorem 10.1. Denote by $A^{D}=\operatorname{Hom}_{\text {cont }}(A, \mathbb{R} / \mathbb{Z})$ the Pontryagin dual of a locally compact abelian group $A$. Let $F$ be a totally imaginary number field and $\mathbb{Z}$ the discrete $W_{F}$-module $\mathbb{Z}$ with trivial action. Then

$$
H^{i}\left(W_{F}, \mathbb{Z}\right)= \begin{cases}\mathbb{Z} & i=0 \\ \left(C_{F}^{1}\right)^{D} & i=2, \\ 0 & i \text { odd }\end{cases}
$$

and $H^{i}\left(W_{F}, \mathbb{Z}\right)$ is an abelian group of infinite rank, in particular nonzero, for even $i \geqslant 4$.

Proof. The idea to compute $H^{i}\left(W_{F}, \mathbb{Z}\right)$ is to use the Hochschild-Serre spectral sequence for the group extension

$$
1 \rightarrow W_{F}^{1,0} \rightarrow W_{F}^{1} \stackrel{\pi}{\rightarrow} G_{F} \rightarrow 1
$$

where

$$
W_{F}^{1,0}=\lim _{K} W_{K / F, S(K)}^{1,0}=\lim _{K} C_{K}^{1,0}
$$

is the connected component of the identity of $W_{F}^{1}$. For any topological group $G$ we denote by $G^{0}$ the connected component of the identity in $G$ which is a closed normal subgroup by [Bou89, III, $\S 2.2$, Proposition 7]. The Hochschild-Serre spectral sequence

$$
H^{j}\left(G_{F}, \underline{H}^{i}\left(W_{F}^{1,0}, \mathbb{Z}\right)\right) \Longrightarrow H^{j+i}\left(W_{F}^{1}, \mathbb{Z}\right)
$$

then exists by Proposition 2.1 and Corollary 6 and computes $H^{j+i}\left(W_{F}, \mathbb{Z}\right)$ by Lemma 10. By Proposition 9.2 we may replace $\mathcal{T}$ by $\mathcal{T}^{l c}$ in this spectral sequence and $\underline{H}^{i}\left(W_{F}^{1,0}, \mathbb{Z}\right)$ is then represented by the discrete $G_{F}$-module $H^{i}\left(W_{F}^{1,0}, \mathbb{Z}\right)$. Alternatively, we may use the Leray spectral sequence for the geometric morphism

$$
p: B W_{F}^{1} \rightarrow B^{s m} W_{F}^{1}=B^{s m} G_{F} .
$$

An argument as in the proof of Proposition 9.4 shows that the sheaf $R^{i} p_{*} \mathbb{Z}$ is the sheaf associated to the presheaf $K \mapsto H_{B W_{F}^{1}}^{i}\left(p^{-1}(K), \mathbb{Z}\right)=H^{i}\left(W_{K}^{1}, \mathbb{Z}\right)$ where $K$ runs through finite extensions of $F$ (the objects of the category $S\left(G_{F}\right)$ in the proof of Proposition 9.4) and $W_{K}^{1}=\pi^{-1}\left(G_{K}\right)$ is the compact Weil group of $K$. The discrete $G_{F}$-module corresponding to $R^{i} p_{*} \mathbb{Z}$ is

$$
M^{i}:=\underset{K}{\lim } H^{i}\left(W_{K}^{1}, \mathbb{Z}\right) \cong H^{i}\left(\bigcap_{K} W_{K}^{1}, \mathbb{Z}\right)=H^{i}\left(W_{F}^{1,0}, \mathbb{Z}\right),
$$

where the first isomorphism follows from Proposition 8.1 and $W_{F}^{1,0}=\bigcap_{K} W_{K}^{1}$ inside $W_{F}^{1}$ since $\{1\}=\bigcap_{K} G_{K}$ inside $G_{F}$.

Again using Proposition 8.1 as well as (16) we find that

$$
M^{i} \cong \underset{K}{\lim _{\longrightarrow}} H^{i}\left(C_{K}^{1,0}, \mathbb{Z}\right) .
$$

For any number field $F$ and Galois extension $K / F$ with group $G$ we have an isomorphism of $G$-spaces

$$
C_{K}^{1,0} \cong \prod_{v \in S_{\infty}, \mathbf{C}(K / F)} \operatorname{Map}_{G_{v}}\left(G, S^{1}\right) \times \mathbb{V}(K),
$$

where $S_{\infty, \mathbf{C}}(K / F)$ is the set of archimedean places of $F$ which become complex in $K$ and $\mathbb{V}(K)$ is a compact group $G$-isomorphic to $\mathbb{V} \otimes_{\mathbb{Z}} \mathcal{O}_{K}^{\times} \cong \mathbb{V}^{r(K)+s(K)-1}$ [NSW00, ch. VIII, Theorem 8.2.5]. Here $r(K)$ (respectively $s(K)$ ) is the number of real (respectively complex) places of $K$ and

$$
\mathbb{V}:=\lim _{n} S^{1} \cong \mathbb{Q}^{D}
$$

is the solenoid. 


\section{FLACH}

LEMma 11. We have

$$
H^{i}(\mathbb{V}(K), \mathbb{Z})= \begin{cases}\mathbb{Z} & i=0 \\ \text { a } \mathbb{Q} \text {-vector space } & i \geqslant 2 \text { even } \\ 0 & i \text { odd }\end{cases}
$$

Moreover $H^{0}\left(G, H^{2}(\mathbb{V}(K), \mathbb{Z})\right)=H^{2}(\mathbb{V}(F), \mathbb{Z})$.

Proof. By Corollary 7 and Proposition 5.2 the cohomology of $\mathbb{V}$ is given by

$$
H^{i}(\mathbb{V}, \mathbb{Z})=\lim _{\longrightarrow} H^{i}\left(S^{1}, \mathbb{Z}\right)= \begin{cases}\mathbb{Z} & i=0, \\ \mathbb{Q} & i \geqslant 2 \text { even }, \\ 0 & i \text { odd },\end{cases}
$$

since the cohomology ring of $\mathbf{B} S^{1} \cong \mathbf{C} P^{\infty}$ is a polynomial ring $\mathbb{Z}[h]$ with a generator $h$ of degree two with $n^{*} h=n h$ where $n^{*}$ is the map induced by multiplication by $n$ on $S^{1}$. The first part of the lemma then follows from the Kuenneth formula of Proposition 8.2. By Proposition 9.6, Corollary 4 and the long exact cohomology sequence induced by the short exact sequence of topological groups with trivial action

$$
0 \rightarrow \mathbb{Z} \rightarrow \mathbb{R} \rightarrow S^{1} \rightarrow 0
$$

we have an isomorphism of functors

$$
\operatorname{Hom}_{\text {cont }}\left(-, S^{1}\right)=H^{1}\left(-, S^{1}\right) \cong H^{2}(-, \mathbb{Z})
$$

on the category of compact groups. Hence there is an isomorphism of $G$-modules

$$
H^{2}(\mathbb{V}(K), \mathbb{Z}) \cong \mathbb{V}(K)^{D} \cong \operatorname{Hom}_{\mathbb{Z}}\left(\mathcal{O}_{K}^{\times}, \mathbb{Q}\right) .
$$

Clearly $\operatorname{Hom}_{\mathbb{Z}}\left(\mathcal{O}_{K}^{\times}, \mathbb{Q}\right)$ has Galois descent which gives the last statement.

If $F$ is totally imaginary then $\operatorname{Map}\left(G, S^{1}\right)=\prod_{g \in G} S^{1}$ and the Kuenneth formula (Proposition 8.2) implies that

$$
R \Gamma\left(C_{K}^{0}, \mathbb{Z}\right) \cong\left(\bigotimes_{v \in S_{\infty}(F)} R \Gamma\left(S^{1}, \mathbb{Z}\right)^{\otimes|G|}\right) \otimes R \Gamma(\mathbb{V}(K), \mathbb{Z})
$$

and a decomposition of $G$-modules

$$
H^{i}\left(C_{K}^{0}, \mathbb{Z}\right)=\bigoplus_{\sum_{v} 2 p_{v}+q=i} \bigotimes_{v \in S_{\infty}(F)} N^{p_{v}}\left(h_{v}\right) \otimes H^{q}(\mathbb{V}(K), \mathbb{Z}),
$$

where

$$
N^{p}(h):=\bigoplus_{i_{1}+\cdots+i_{g}=p} \mathbb{Z} \cdot h_{1}^{i_{1}} \cdots h_{g}^{i_{g}}
$$

is the permutation module on the set of monomials of degree $p$ on the variables $h_{1}, \ldots, h_{g}$ indexed by $G$. Note here that

$$
R \Gamma\left(S^{1}, \mathbb{Z}\right)^{\otimes|G|} \cong R \Gamma\left(\mathbf{B} S^{1}, \mathbb{Z}\right)^{\otimes|G|} \cong \mathbb{Z}\left[h_{1}, \ldots, h_{g}\right] .
$$

From Lemma 11 we deduce that

$$
H^{0}\left(G, H^{i}\left(C_{K}^{1,0}, \mathbb{Z}\right)\right)=\bigoplus_{\sum_{v} 2 p_{v}+q=i} H^{0}\left(G, \bigotimes_{v \in S_{\infty}(F)} N^{p_{v}}\left(h_{v}\right) \otimes H^{q}(\mathbb{V}(K), \mathbb{Z})\right)
$$

and

$$
H^{j}\left(G, H^{i}\left(C_{K}^{1,0}, \mathbb{Z}\right)\right)=\bigoplus_{\sum_{v} 2 p_{v}=i} H^{j}\left(G, \bigotimes_{v \in S_{\infty}(F)} N^{p_{v}}\left(h_{v}\right)\right)
$$




\section{COHOMOLOGY OF TOPOLOGICAL GROUPS}

for $j \geqslant 1$, as $G$ is finite and $H^{q}(\mathbb{V}(K), \mathbb{Z})$ a $\mathbb{Q}$-vector space for $q>0$. Moreover

$$
H^{0}\left(G, H^{2}\left(C_{K}^{1,0}, \mathbb{Z}\right)\right) \cong \bigoplus_{v \in S_{\infty}(F)} \mathbb{Z} \oplus H^{2}(\mathbb{V}(F), \mathbb{Z}) \cong \mathbb{Z}^{S_{\infty}(F)} \times \mathbb{V}(F)^{D} \cong\left(C_{F}^{1,0}\right)^{D} .
$$

The $E^{2}$-term of the Leray spectral sequence for the morphism $p: B W_{F}^{1} \rightarrow B^{s m} G_{F}$,

$$
\underset{K}{\lim _{\longrightarrow}} H^{j}\left(\operatorname{Gal}(K / F), H^{i}\left(C_{K}^{1,0}, \mathbb{Z}\right)\right)=H^{j}\left(G_{F}, M^{i}\right) \Rightarrow H^{j+i}\left(W_{F}^{1}, \mathbb{Z}\right),
$$

or equivalently of the Hochschild-Serre spectral sequence (17), therefore looks like

$$
\begin{array}{cccc}
E_{04}^{2} & 0 & E_{24}^{2} & 0 \\
0 & 0 & 0 & 0 \\
\left(C_{F}^{1,0}\right)^{D} & 0 & 0 & 0 \\
0 & 0 & 0 & 0 \\
\mathbb{Z} & 0 & \left(G_{F}^{a b}\right)^{D} & 0
\end{array}
$$

where the vanishing entries are given by the following lemma.

LEMma 12. The following entries in (24) vanish.

(a) All rows with odd index.

(b) The row with index 2 after the first entry.

(c) The column with index 1 .

(d) All columns with index 3 or more.

Proof. Item (a) follows since $R \Gamma\left(C_{K}^{1,0}, \mathbb{Z}\right)$ is concentrated in even degrees by (21) and Lemma 11. Concerning (b) we have

$$
H^{j}\left(G, H^{2}\left(C_{K}^{1,0}, \mathbb{Z}\right)\right)=\bigoplus_{v \in S_{\infty}(F)} H^{j}\left(G, N^{1}\left(h_{v}\right)\right)=0
$$

for $j \geqslant 1$ because $N^{1}(h) \cong \mathbb{Z}[G]$ is $\mathbb{Z}[G]$-projective, hence cohomologically trivial. For any tuple $\left(p_{v}\right)$ the coefficient module $\bigotimes_{v \in S_{\infty}(F)} N^{p_{v}}\left(h_{v}\right)$ is a permutation module, i.e. the free abelian group $\mathbb{Z}[S]$ on a $G$-set $S$. For such a module $H^{0}(G, \mathbb{Z}[S])$ is the free abelian group on the set of $G$-orbits and $H^{0}(G, A[S])=H^{0}(G, \mathbb{Z}[S]) \otimes_{\mathbb{Z}} A$ for any abelian group $A$. Hence $H^{0}(G,-)$ applied to the short exact sequence

$$
0 \rightarrow \mathbb{Z}[S] \rightarrow \mathbb{Q}[S] \rightarrow \mathbb{Q} / \mathbb{Z}[S] \rightarrow 0
$$

gives an exact sequence. Since $\mathbb{Q}[S]$ is uniquely divisible and $G$ finite we have $H^{1}(G, \mathbb{Q}[S])=0$ and hence $H^{1}(G, \mathbb{Z}[S])=0$. This gives item (c). Finally (d) follows from the fact that the strict cohomological dimension of the Galois group $G_{F}$ of the totally imaginary field $F$ is 2 [Mil86, Remark 1.12].

It follows that all differentials in (24) vanish, that $E^{2}=E^{\infty}$ and that $H^{i}\left(W_{F}, \mathbb{Z}\right)=0$ for $i$ odd. The computation of $H^{2}\left(W_{F}, \mathbb{Z}\right)$ is $(20)$ combined with Lemma 10 since $W_{F}^{1, a b} \cong C_{F}^{1}$. There is a surjection $H^{i}\left(W_{F}, \mathbb{Z}\right) \rightarrow E_{0 i}^{2}=H^{0}\left(G_{F}, H^{i}\left(W_{F}^{1,0}, \mathbb{Z}\right)\right)$ and it remains to show that $H^{0}\left(G_{F}, H^{i}\left(W_{F}^{1,0}, \mathbb{Z}\right)\right)$ is an abelian group of infinite rank for even $i \geqslant 4$. Consider the summand in (23) corresponding to $q=0, p_{w}=i / 2$ for exactly one $w \in S_{\infty}(F)$ and $p_{v}=0$ for $v \neq w$ which is isomorphic to

$$
H^{0}\left(G, \bigoplus_{w \in S_{\infty}(F)} N^{i / 2}\left(h_{w}\right)\right) \text {. }
$$




\section{FLACH}

In the formula (22) for $p=i / 2$ consider the $G$-submodule

$$
\mathbb{Z}\left[\Sigma_{p}(G)\right]=\bigoplus_{\left\{g_{1}, \ldots, g_{p}\right\} \subseteq G} \mathbb{Z} \cdot h_{g_{1}} h_{g_{2}} \cdots h_{g_{p}}
$$

of polynomials with all exponents equal to 1 which is isomorphic to the permutation module on the set $\Sigma_{p}(G)$ of subsets of order $p$ of $G$. This set has cardinality $\left(\begin{array}{c}|G| \\ p\end{array}\right)$ and the number $r(G, p)$ of its orbits is at least $\left(\begin{array}{c}|G| \\ p\end{array}\right) /|G|$. Since $p>1$ the group

$$
H^{0}\left(G, \bigoplus_{\left\{g_{1}, \ldots, g_{p}\right\} \subseteq G} \mathbb{Z} \cdot h_{g_{1}} h_{g_{2}} \cdots h_{g_{p}}\right) \cong \mathbb{Z}^{r(G, p)}
$$

has rank tending to infinity as $|G|$ tends to infinity, hence the same is true for (25). If $K^{\prime} / F$ is a Galois extension containing $K$ and with group $G^{\prime}$, the surjection $\pi: G^{\prime} \rightarrow G$ induces a $G^{\prime}$ homomorphism $N^{p}(h) \rightarrow N^{p}\left(h^{\prime}\right)$ sending $h_{g}$ to $\sum_{\pi\left(g^{\prime}\right)=g} h_{g^{\prime}}^{\prime}$. This homomorphism is injective and hence so is

$$
H^{0}\left(G, \bigoplus_{w \in S_{\infty}(F)} N^{i / 2}\left(h_{w}\right)\right) \rightarrow H^{0}\left(G^{\prime}, \bigoplus_{w \in S_{\infty}(F)} N^{i / 2}\left(h_{w}^{\prime}\right)\right) .
$$

This implies that the abelian groups

$$
\underset{K}{\lim _{K}} H^{0}\left(G, \bigoplus_{w \in S_{\infty}(F)} N^{i / 2}\left(h_{w}\right)\right)
$$

and

$$
H^{0}\left(G_{F}, H^{i}\left(W_{F}^{1,0}, \mathbb{Z}\right)\right)=\underset{K}{\lim _{\longrightarrow}} H^{0}\left(G, H^{i}\left(C_{K}^{1,0}, \mathbb{Z}\right)\right)
$$

are of countably infinite rank.

Remark. By analyzing the cohomology of $\mathbb{V}(K)$ one can also produce a $\mathbb{Q}$-vector space (likely of infinite rank) in $H^{i}\left(W_{F}, \mathbb{Z}\right)$ for even $i \geqslant 4$.

\section{The global Weil-étale topology}

Let $Y=\operatorname{Spec}\left(\mathcal{O}_{F}\right)$ be the ring of integers in the number field $F$. In this section we briefly record the consequences of Theorem 10.1 for the Weil-étale cohomology groups $H^{i}\left(\bar{Y}_{W}, \mathbb{Z}\right)$ and $H_{c}^{i}\left(Y_{W}, \mathbb{Z}\right)$ defined by Lichtenbaum in [Lic04], following his method of using the Leray spectral sequence of the inclusion of the generic point.

For any nontrivial absolute value $v$ of $F$, we denote by $W_{v}$ the Weil group of the local field $F_{v}$ and by $W_{\kappa(v)} \cong \mathbb{Z}$ the Weil group of the residue field if $v$ is nonarchimedean. In the case where $v$ is archimedean we let $W_{\kappa(v)}$ be the canonical quotient $\mathbb{R}$ of $W_{v}$ given by the logarithm of the absolute value map. For all $v$ there is a short exact sequence of topological groups

$$
0 \rightarrow W_{v}^{1} \rightarrow W_{v} \rightarrow W_{\kappa(v)} \rightarrow 0,
$$

where $W_{v}^{1}$ is the maximal compact subgroup of $W_{v}$. Hence $W_{v}^{1}=I_{v}$ is the inertia subgroup for nonarchimedean $v$ and $W_{v}^{1} \cong S^{1}$ for complex $v$.

TheOREM 11.1. Let $F$ be a totally imaginary number field. Then the abelian groups $H^{i}\left(\bar{Y}_{W}, \mathbb{Z}\right)$ and $H_{c}^{i}\left(Y_{W}, \mathbb{Z}\right)$ are of infinite rank for even $i \geqslant 4$ and vanish for odd $i \geqslant 5$.

Proof. By [Lic04, Theorem 4.7] there is a spectral sequence

$$
H^{p}\left(\bar{Y}_{W}, R^{q} j_{*} \mathbb{Z}\right) \Longrightarrow H^{p+q}\left(W_{F}, \mathbb{Z}\right)
$$




\section{COHOMOLOGY OF TOPOLOGICAL GROUPS}

which is in fact a direct limit over $K$ and finite sets $S$ of Leray spectral sequences for morphisms of topoi $j_{K, S}: B W_{K / F, S} \rightarrow \bar{Y}_{K, S, W}$ where $\bar{Y}_{K, S, W}$ is the category of sheaves of sets for the site $T_{K, S, \bar{Y}}$ defined in [Lic04, $\S 4]$. By [Lic04, Corollary 4.12] there is an isomorphism

$$
H^{p}\left(\bar{Y}_{W}, R^{q} j_{*} \mathbb{Z}\right) \cong \bigoplus_{v} H^{p}\left(W_{\kappa(v)}, i_{v}^{*} R^{q} j_{*} \mathbb{Z}\right)
$$

for $q>0$ where the sum is over all places $v$ of $F$.

Lemma 13. For an abelian group object $\mathcal{A}$ of $B W_{K / F, S}$ and any place $v$ of $F$ the objects $i_{v}^{*} R^{q} j_{K, S, *} \mathcal{A}$ of $B W_{\kappa(v)}$ are given by $\underline{H}^{q}\left(\tilde{W}_{v}^{1}, \mathcal{A}\right)$ where $\tilde{W}_{v}^{1}$ is the image of $W_{v}^{1}$ under the homomorphism $W_{v} \rightarrow$ $W_{K / F, S}$.

Proof. Recall that $T_{K, S, \bar{Y}}$ is a site with objects $\left(X_{v}, X_{v_{0}}, f_{v}\right)$ where $X_{v}$ (respectively $\left.X_{v_{0}}\right)$ is an object of $B_{\text {Top }} W_{\kappa(v)}$ (respectively $B_{\text {Top }} W_{K / F, S}$ ) and $f_{v}: X_{v} \rightarrow X_{v_{0}}$ a map of $W_{v}$-spaces. Coverings are local section covers in each component. The sheaf $j_{K, S, *} \mathcal{A}$ is given by

$$
j_{K, S, *} \mathcal{A}\left(X_{v}, X_{v_{0}}, f_{v}\right)=\mathcal{A}\left(X_{v_{0}}\right) .
$$

Fix a place $w$. The sheaf $i_{w}^{*} j_{K, S, * \mathcal{A}}$ is the sheaf associated to the presheaf

$$
Z \mapsto \lim _{\longrightarrow}\left(j_{K, S, * \mathcal{A}}\right)\left(X_{v}, X_{v_{0}}, f_{v}\right)=\lim _{\longrightarrow} \mathcal{A}\left(X_{v_{0}}\right),
$$

where the limit is over the category with objects $\left(X_{v}, X_{v_{0}}, f_{v}, g\right)$ where $g: Z \rightarrow X_{w}$ is a map in $B_{\text {Top }} W_{\kappa(w)}$. This category has the initial object $\left(Z_{v}, Z_{v_{0}}, \phi_{v}, \mathrm{id}_{Z}\right)$ where $Z_{w}=Z, Z_{v}=\emptyset$ for $v \neq w$, $Z_{v_{0}}=E W_{K / F, S} \times_{W_{w}} Z$ and $\phi_{w}: Z \rightarrow E W_{K / F, S} \times_{W_{w}} Z$ the inclusion. Here $E W_{K / F, S} \times_{W_{w}} Z$ is the quotient of $E W_{K / F, S} \times Z$ by the diagonal action of $W_{w}$ and the fact that this object is initial follows from the adjunction

$$
\operatorname{Hom}_{B_{\mathrm{Top}} W_{K / F, S}}\left(E W_{K / F, S} \times_{W_{w}} Z, X\right) \cong \operatorname{Hom}_{B_{\mathrm{Top}} W_{w}}(Z, X) .
$$

By Corollary 3 we may take as a defining site for $B W_{\kappa(w)}$ the category of all $W_{\kappa(w)}$-spaces $Z=$ $W_{\kappa(w)} \times Z^{\prime}$ with a topological space $Z^{\prime}$. Denoting by $y$ : Top $\rightarrow \mathcal{T}$ the Yoneda embedding we have

$$
\begin{aligned}
y\left(E W_{K / F, S} \times_{W_{w}} Z\right) & =y\left(E W_{K / F, S} \times_{W_{w}} W_{\kappa(w)} \times Z^{\prime}\right) \\
& =y\left(E W_{K / F, S}\right) \times_{y W_{w}} y W_{\kappa(w)} \times y Z^{\prime}=y\left(E W_{K / F, S}\right) \times_{y W_{w}} y Z,
\end{aligned}
$$

where the second identity follows from Proposition 2.1. Hence $i_{w}^{*} j_{K, S, *} \mathcal{A}$ is the sheaf associated to the presheaf

$$
\begin{aligned}
Z & \mapsto \mathcal{A}\left(E W_{K / F, S} \times_{W_{w}} Z\right)=\operatorname{Hom}_{B W_{K / F, S}}\left(y\left(E W_{K / F, S} \times_{W_{w}} Z\right), \mathcal{A}\right) \\
& =\operatorname{Hom}_{B W_{w}}\left(W_{\kappa(w)} \times Z^{\prime}, \mathcal{A}\right)=\operatorname{Hom}_{\mathcal{T}}\left(Z^{\prime}, \underline{\operatorname{Hom}}_{W_{w}}\left(W_{\kappa(w)}, \mathcal{A}\right)\right) \\
& =\operatorname{Hom}_{\mathcal{T}}\left(Z^{\prime}, \underline{\operatorname{Hom}}_{W_{w}^{1}}(*, \mathcal{A})\right)=\operatorname{Hom}_{B W_{\kappa(w)}}\left(Z, \underline{\operatorname{Hom}}_{W_{w}^{1}}(*, \mathcal{A})\right),
\end{aligned}
$$

where the last (respectively second to last) identity is Lemma 7(a) (respectively equation (8)). We conclude that this presheaf is already a sheaf given by

$$
i_{w}^{*} j_{K, S, *} \mathcal{A}=\underline{\operatorname{Hom}}_{W_{w}^{1}}(*, \mathcal{A})=\underline{\operatorname{Hom}}_{\tilde{W}_{w}^{1}}(*, \mathcal{A})=e_{\tilde{W}_{w}^{1}, *} \mathcal{A} .
$$

Since $\tilde{W}_{w}^{1} \rightarrow W_{K / F, S}$ is a subgroup, the restriction functor $A b\left(B W_{K / F, S}\right) \rightarrow A b\left(B \tilde{W}_{w}^{1}\right)$ sends injective objects to injective objects and hence the right derived functors of (28) are the objects $\underline{H}^{q}\left(\tilde{W}_{w}^{1}, \mathcal{A}\right):=R^{q} e \tilde{W}_{w}^{1} \mathcal{A}$ of $B W_{k(w)}$. Since $i_{w}^{*}$ is exact these agree with $i_{w}^{*} R^{q} j_{K, S, *} \mathcal{A}$.

Corollary 9 (See also [Lic04, Lemma 5.2] for nonarchimedean v.). For any place $v$ of $F$ we have

$$
H^{p}\left(W_{\kappa(v)}, i_{v}^{*} R^{q} j_{*} \mathbb{Z}\right) \cong H^{p}\left(W_{\kappa(v)}, H^{q}\left(W_{v}^{1}, \mathbb{Z}\right)\right) .
$$




\section{FLACH}

Proof. By definition

$$
H^{p}\left(W_{\kappa(v)}, i_{v}^{*} R^{q} j_{*} \mathbb{Z}\right)=\underset{K, S}{\lim _{K, S}} H^{p}\left(W_{\kappa(v)}, i_{v}^{*} R^{q} j_{K, S, *} \mathbb{Z}\right)=\underset{K, S}{\lim _{K,}} H^{p}\left(W_{\kappa(v)}, \underline{H}^{q}\left(\tilde{W}_{v}^{1}, \mathbb{Z}\right)\right) .
$$

By Proposition 9.2 the sheaves $\underline{H}^{q}\left(\tilde{W}_{v}^{1}, \mathbb{Z}\right)$ are represented by the discrete $W_{\kappa(v)}$-module $H^{q}\left(\tilde{W}_{v}^{1}, \mathbb{Z}\right)$. Using Propositions 8.1 and 9.6 we find that

$$
\underset{K, S}{\lim _{K, S}} H^{p}\left(W_{\kappa(v)}, \underline{H}^{q}\left(\tilde{W}_{v}^{1}, \mathbb{Z}\right)\right)=H^{p}\left(W_{\kappa(v)}, H^{q}\left(W_{v}^{1}, \mathbb{Z}\right)\right) .
$$

In this computation we have maintained compatibility with the definitions of [Lic04] (and our results are only slightly more general in that they cover archimedean $v$ ). One could instead define a site $T_{\bar{Y}}$ with objects $\left(X_{v}, X_{v_{0}}, f_{v}\right)$ where $X_{v_{0}}$ is a $W_{F}$-space, obtain a Leray spectral sequence (26), prove Lemma 13 in the same way with $W_{K / F, S}$ and $\tilde{W}_{v}$ replaced by $W_{F}$ and $W_{v}$, respectively, and end up with the same computation of $H^{i}\left(\bar{Y}_{W}, \mathbb{Z}\right)$ (where the analog of $(27)$ remains to be verified, however).

For nonarchimedean $v$, by [Ser65, ch. I, Proposition 13, ch. II, Proposition 12], the strict cohomological dimension of $W_{v}^{1}=I_{v}$ is 2 and

$$
H^{q}\left(I_{v}, \mathbb{Z}\right)= \begin{cases}\mathbb{Z} & q=0 \\ \left(G_{F_{v}^{u r}}^{a b}\right)^{D} & q=2 \\ 0 & q \neq 0,2\end{cases}
$$

and therefore by class field theory and the fact that $\left(G_{F_{v}^{u r}}^{a b}\right)^{D}$ is divisible,

$$
H^{p}\left(W_{\kappa(v)}, H^{q}\left(I_{v}, \mathbb{Z}\right)\right)= \begin{cases}\mathbb{Z} & p \in\{0,1\}, q=0, \\ \left(\mathcal{O}_{F_{v}}^{\times}\right)^{D} & p=0, q=2, \\ 0 & \text { otherwise. }\end{cases}
$$

For archimedean $v$ we have $W_{v} \cong \mathbb{C}^{\times}, W_{v}^{1} \cong S^{1}$,

$$
H^{q}\left(W_{v}^{1}, \mathbb{Z}\right) \cong H^{q}\left(W_{v}, \mathbb{Z}\right) \cong \begin{cases}\mathbb{Z} & q \text { even }, \\ 0 & q \text { odd },\end{cases}
$$

and therefore by Proposition 9.6

$$
H^{p}\left(W_{\kappa(v)}, H^{q}\left(W_{v}^{1}, \mathbb{Z}\right)\right)= \begin{cases}\mathbb{Z} & p=0 \text { and } q \text { even } \\ 0 & \text { otherwise. }\end{cases}
$$

The spectral sequence (26) therefore looks as follows:

$$
\begin{array}{ccccc}
\bigoplus_{v \mid \infty} \mathbb{Z} & 0 & 0 & 0 & 0 \\
0 & 0 & 0 & 0 & 0 \\
\bigoplus_{v \nmid \infty}\left(\mathcal{O}_{F_{v}}^{\times}\right)^{D} \oplus \bigoplus_{v \mid \infty} \mathbb{Z} & 0 & 0 & 0 & 0 \\
0 & 0 & 0 & 0 & 0 \\
H^{0}\left(\bar{Y}_{W}, \mathbb{Z}\right) & H^{1}\left(\bar{Y}_{W}, \mathbb{Z}\right) & H^{2}\left(\bar{Y}_{W}, \mathbb{Z}\right) & H^{3}\left(\bar{Y}_{W}, \mathbb{Z}\right) & H^{4}\left(\bar{Y}_{W}, \mathbb{Z}\right)
\end{array}
$$

Using Theorem 10.1 we recover Lichtenbaum's computation of $H^{i}\left(\bar{Y}_{W}, \mathbb{Z}\right)$ for $i \leqslant 3$ in [Lic04, Theorem 5.10] and an exact sequence

$$
0 \rightarrow H^{i}\left(\bar{Y}_{W}, \mathbb{Z}\right) \rightarrow H^{i}\left(W_{F}, \mathbb{Z}\right) \stackrel{\rho}{\rightarrow} \bigoplus_{v \in S_{\infty}(F)} \mathbb{Z} \rightarrow H^{i+1}\left(\bar{Y}_{W}, \mathbb{Z}\right) \rightarrow 0
$$

for even $i \geqslant 4$. Hence for such $i$ the group $H^{i}\left(\bar{Y}_{W}, \mathbb{Z}\right)$ is of infinite rank. For archimedean $v$ the 


\section{COHOMOLOGY OF TOPOLOGICAL GROUPS}

morphisms of groups $W_{v}^{1} \rightarrow W_{F}^{0} \rightarrow C_{F}^{0}$ induce the following commutative diagram.

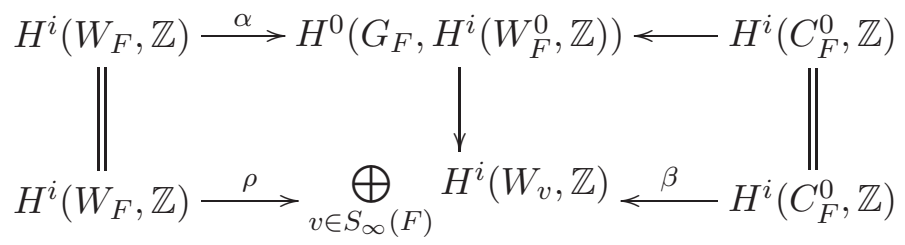

The proof of Theorem 10.1 shows that the map $\alpha$ is surjective, and the map $\beta$ is surjective by the Kuenneth formula and the fact that $W_{v}^{1} \rightarrow C_{F}^{0}$ is the inclusion of the factor corresponding to $v$ in (18) for $K=F$. Hence $\rho$ is surjective and therefore $H^{i+1}\left(\bar{Y}_{W}, \mathbb{Z}\right)=0$ for even $i \geqslant 4$. The long exact sequence

$$
\cdots \rightarrow H_{c}^{i}\left(Y_{W}, \mathbb{Z}\right)=H^{i}\left(\bar{Y}_{W}, \phi_{!} \mathbb{Z}\right) \rightarrow H^{i}\left(\bar{Y}_{W}, \mathbb{Z}\right) \rightarrow \bigoplus_{v \in S_{\infty}(F)} H^{i}\left(W_{\kappa(v)}, \mathbb{Z}\right) \rightarrow \cdots
$$

induced by the short exact sequence [Lic04, Proposition 6.1] together with the vanishing of $H^{i}\left(W_{\kappa(v)}, \mathbb{Z}\right)$ for $i>0$ (Proposition 9.6 or [Lic04, Lemma 6.2]) then shows that $H_{c}^{i}\left(Y_{W}, \mathbb{Z}\right) \cong$ $H^{i}\left(\bar{Y}_{W}, \mathbb{Z}\right)$ for $i \geqslant 2$.

\section{ACKNowledgements}

I am very grateful to Steve Lichtenbaum for extremely stimulating discussions about Weil-étale cohomology and for inviting me to speak at Brown University. I would also like to thank Thomas Geisser for many discussions related to topos theory, in particular concerning Proposition 7.1 above.

\section{REFERENCES}

BW00 A. Borel and N. Wallach, Continuous cohomology, discrete subgroups and representations of reductive groups, second edition (American Mathematical Society, Providence, RI, 2000).

Bou89 N. Bourbaki, General topology (Springer, Berlin, 1989).

Bre97 G. E. Bredon, Sheaf theory, second edition, Graduate Texts in Mathematics, vol. 170 (Springer, New York, 1997).

Dia75 R. Diaconescu, Change of base for toposes with generators, J. Pure Appl. Math. 6 (1975), 191-218.

EM47 S. Eilenberg and S. MacLane, Cohomology theory in abstract groups I, Ann. of Math. (2) 48 (1947), 51-78.

HM62 G. P. Hochschild and G. D. Mostow, Cohomology of Lie groups, Illinois J. Math. 6 (1962), 367-401.

Lic04 S. Lichtenbaum, The Weil-étale topology for number rings, Preprint (2004), Ann. of Math, to appear.

MM92 S. Maclane and I. Moerdijk, Sheaves in geometry and logic (Universitext, Springer, Berlin, 1992).

Mic56 E. Michael, Continuous selections II, Ann. of Math. (2) 64 (1956), 562-580.

Mil86 J. S. Milne, Arithmetic duality theorems, Perspectives in Mathematics, vol. 1 (Academic Press, New York, 1986).

Moe95 I. Moerdijk, Classifying spaces and classifying topoi, Lecture Notes in Mathematics, vol. 1616 (Springer, Berlin, 1995).

Moo64 C. C. Moore, Extensions and low dimensional cohomology theory of locally compact groups I, II, Trans. Amer. Math. Soc. 113 (1964), 40-63, 64-86.

NSW00 J. Neukirch, A. Schmidt and K. Wingberg, Cohomology of number fields, Grundlehren der mathematischen Wissenschaften, vol. 323 (Springer, Berlin, 2000).

Raj04 C. S. Rajan, On the vanishing of the measurable Schur cohomology groups of Weil groups, Compositio Math. 140 (2004), 84-98. 


\section{COHOMOLOGY OF TOPOLOGICAL GROUPS}

Seg70 G. Segal, Cohomology of topological groups, in Istituto Nazionale di alta matematica, Symposia Mathematica, vol. IV (Academic Press, New York, 1970).

Ser49 J. P. Serre, Extensions de groupes localement compacts (d'apres Iwasawa et Gleason), Seminaire Bourbaki, Exposé 27, 1949/50.

Ser65 J. P. Serre, Cohomologie Galoisienne, Lecture Notes in Mathematics, vol. 5 (Springer, Berlin, 1965).

SGA3 A. Grothendieck and M. Demazure, Schemas en groupes (SGA3), Lecture Notes in Mathematics, vols. 151, 152, 153 (Springer, Berlin, 1970).

SGA4 A. Grothendieck, M. Artin and J. L. Verdier, Theorie des topos et cohomologie étale des schemas (SGA4), Lecture Notes in Mathematics. vols. 269, 270, 271 (Springer, Berlin, 1972).

vEs53 W. T. van Est, Group cohomology and Lie algebra cohomology in Lie groups I, II, Nederl. Akad. Wetensch. Proc. Ser. A. 56 = Indag. Math. 15 (1953), 484-504.

Wei51 A. Weil, L'intégration dans les groupes topologiques et ses applications (Hermann, Paris, 1951).

Wig73 D. Wigner, Algebraic cohomology of topological groups, Trans. Amer. Math. Soc. 178 (1973), 83-93.

M. Flach flach@its.caltech.edu

Department of Mathematics, Caltech, Pasadena, CA 91125, USA 